\title{
Radio synchrotron spectra of star-forming galaxies
}

\author{
U. Klein ${ }^{1}$, U. Lisenfeld ${ }^{2,3}$, and S. Verley ${ }^{2,3}$ \\ 1 AIfA, Universität Bonn, Auf dem Hügel 71, 53121 Bonn, Germany \\ e-mail: uklein@astro.uni-bonn.de \\ 2 Departamento de Física Teórica y del Cosmos, Universidad de Granada, 18010 Granada, Spain \\ e-mail: [ute; simon]@ugr.es \\ ${ }^{3}$ Instituto Universitario Carlos I de Física Teórica y Computacional, Facultad de Ciencias, 18071 Granada, Spain \\ Received 30 July 2017 / Accepted 8 October 2017
}

\begin{abstract}
We investigated the radio continuum spectra of 14 star-forming galaxies by fitting nonthermal (synchrotron) and thermal (free-free) radiation laws. The underlying radio continuum measurements cover a frequency range of $\sim 325 \mathrm{MHz}$ to $24.5 \mathrm{GHz}$ ( $32 \mathrm{GHz}$ in case of M 82). It turns out that most of these synchrotron spectra are not simple power-laws, but are best represented by a low-frequency spectrum with a mean slope $\alpha_{\text {nth }}=0.59 \pm 0.20\left(S_{v} \propto v^{-\alpha}\right)$, and by a break or an exponential decline in the frequency range of 1-12 GHz. Simple power-laws or mildly curved synchrotron spectra lead to unrealistically low thermal flux densities, and/or to strong deviations from the expected optically thin free-free spectra with slope $\alpha_{\mathrm{th}}=0.10$ in the fits. The break or cutoff energies are in the range of 1.5-7 GeV. We briefly discuss the possible origin of such a cutoff or break. If the low-frequency spectra obtained here reflect the injection spectrum of cosmic-ray electrons, they comply with the mean spectral index of Galactic supernova remnants. A comparison of the fitted thermal flux densities with the (foreground-corrected) $\mathrm{H} \alpha$ fluxes yields the extinction, which increases with metallicity. The fraction of thermal emission is higher than believed hitherto, especially at high frequencies, and is highest in the dwarf galaxies of our sample, which we interpret in terms of a lack of containment in these low-mass systems, or a time effect caused by a very young starburst.
\end{abstract}

Key words. acceleration of particles - radiation mechanisms: non-thermal - radio continuum: galaxies - galaxies: magnetic fields galaxies: star formation

\section{Introduction}

Star-forming galaxies emit both thermal (free-free) and nonthermal (synchrotron) radiation in the radio regime. It has been known for a few decades that the synchrotron component dominates at frequencies up to about $10 \mathrm{GHz}$ (Klein \& Emerson 1981; Gioia et al. 1982) and that the overall slope of the observed spectra has a mean value of about 0.70 to 0.75 , with little dispersion (about 0.10). Furthermore, for decades the perception has been that the spectra are the superposition of two power-laws,

$S_{\text {tot }}(v)=S_{\text {th }}\left(v_{0}\right)\left(\frac{v}{v_{0}}\right)^{-0.1}+S_{\text {nth }}\left(v_{0}\right)\left(\frac{v}{v_{0}}\right)^{-\alpha_{\text {nth }}}$,

where $\alpha_{\text {nth }}$ is the spectral index of the synchrotron radiation spectrum. The superposition of thermal and synchrotron radiation thus produces a flattening of the total radio spectrum towards high frequencies, with the asymptotic value of -0.1 . This, however, can hardly be seen, owing to the onset of thermal radiation from dust, which becomes significant above about $40 \mathrm{GHz}$.

In the past decade, numerous studies have been dedicated to characterising the shape of the radio spectrum. The two major questions have been: What is the contribution of the thermal emission (thermal fraction, $f_{\mathrm{th}}=S_{\mathrm{th}} / S_{\text {tot }}$ )? What is the synchrotron spectral index, $\alpha_{\text {nth }}$, and how does it change with frequency? Past studies have given different answers, owing to the differences in the covered frequency range and the sample selection. Gioia et al. (1982) found a mean spectral index of the total radio continuum emission of $\alpha_{\text {tot }} \approx 0.7$, based on a sample of 56 galaxies, which they had observed at $0.408,4.75$ and
10.7 GHz. They concluded that the spectral index of the synchrotron radiation is $\alpha_{\text {syn }} \approx 0.8$, with the synchrotron emission dominating below $10 \mathrm{GHz}$. They estimated the fraction of thermal radiation to be $f_{\text {th } 10 \mathrm{GHz}}<40 \%$. Klein (1988) found a mean value of $\alpha_{\text {nth }}=0.88 \pm 0.06$, and a fraction of thermal emission of $\$ 40 \%$ at $10 \mathrm{GHz}$. Duric et al. (1988), however, claimed a large variation of the thermal fraction in a sample of 41 spiral galaxies. Niklas et al. (1997) found a thermal fraction $f_{\mathrm{th}, 1 \mathrm{GHz}}=8 \% \pm 1 \%$, based upon observations of a sample of 74 Shapley-Ames galaxies at $10.7 \mathrm{GHz}$. Williams \& Bower (2010) obtained radio continuum spectra of galaxies with continuous frequency coverage between 1 and $7 \mathrm{GHz}$. They found curved synchrotron spectra for the starburst galaxies NGC 253, M 82, and Arp 220. Marvil et al. (2015) presented radio continuum spectra of 250 bright galaxies, reporting a curvature of the spectra between $74 \mathrm{MHz}$ and $4.85 \mathrm{GHz}$, with $\alpha_{\text {nth }}$ changing from 0.45 to 0.69 . These latter two papers strongly indicate that the synchrotron spectra of star-forming galaxies cannot be simple power-laws, but probably decline with increasing frequency in the cm-wavelength range. Recently, Tabatabaei et al. (2017) presented measurements of 61 galaxies at 1.4, 4.8, 8.5, and $10.5 \mathrm{GHz}$. Their analysis delivered a total spectral index of $\alpha_{\text {tot }}=0.79 \pm 0.15$, a mean synchrotron spectral index of $\alpha_{\text {nth }}=0.97 \pm 0.16$, and a fraction of thermal radiation of $f_{\text {th, } 1 \mathrm{GHz}}=10 \% \pm 9 \%$.

There are several reasons that may have led to the variety of the derived synchrotron spectra and thermal fractions summarised above, some of which are contradicting. First, the quality of early $(\$ 1990)$ continuum measurements of galaxies has 
been inferior to that of observations conducted thereafter. Second, the sample sizes used in the analyses were rather different, and the frequency coverage of the measurements culled from the literature too small. Third, the careful data selection described in Sect. 3 may not have been made in some of the works.

The spectrum of the diffuse synchrotron emission is shaped by the processes that characterise the propagation of relativistic electrons, in particular the type of propagation (diffusion or convection), of energy losses (synchrotron, inverse-Compton, adiabatic losses or bremsstrahlung), and the confinement to the galaxy. It is generally accepted that the acceleration takes place in the shocks of supernova remnants (SNRs), which have an average spectral index of -0.5 (Green 2014). From there, the relativistic particles propagate into the interstellar medium (ISM) via diffusion and/or convection, and lose energy. The dominant energy losses at high frequencies are synchrotron and inverse-Compton losses, which steepen the synchrotron spectrum. At low frequencies thermal absorption would flatten the spectra and may even produce a turn-over at the lowest frequencies (see the papers and discussion by Israel \& Mahoney 1990; Hummel 1991). However, for the frequency range considered in the present paper, we are not concerned with this latter process, as it would require extreme emission measures in order to become relevant for the frequency range considered here.

In this paper, we present an analysis of accurately determined radio continuum spectra of 14 star-forming galaxies, comprising massive grand-design, closely interacting, and lowmass (dwarf) galaxies. The spectra cover a frequency range $\sim 325 \mathrm{MHz}-24.5 \mathrm{GHz}$ (32 GHz in case of M 82), with the $24.5 \mathrm{GHz}$ measurements performed with the Effelsberg 100-m telescope in the mid 1980s, and mostly unpublished to date. All other flux densities were carefully selected from the literature. For the first time, the data allow a reliable separation of the thermal and nonthermal components to be performed, and hence an analysis of the resulting synchrotron spectra, and a firm extrapolation towards lower frequencies. This kind of work is also considered valuable towards establishing and interpreting low-frequency spectra of galaxies obtained with LOFAR observations (see, e.g. Mulcahy et al. 2014). However, for this paper we decided not to use measurements at frequencies below $325 \mathrm{MHz}$ since astrophysical processes that shape the spectra there would unnecessarily increase the number of free parameters.

In Sect. 2 we present the galaxy sample and their selection criteria. The data selection made for the present investigation is described in Sect. 3. In Sect. 4 the fitting procedure of the spectra is described, and in Sect. 5 the results are presented. These results are discussed in Sect. 6, while Sect. 7 contains a summary and lists our conclusions.

\section{The galaxies}

The galaxy sample used for our analysis was determined by the inclusion of high-frequency radio continuum data (see Sect. 3.2), which are available for a small number of galaxies mapped with the Effelsberg 100-m telescope years ago. This results in a mixture of rather different galaxy types, spanning the whole mass range of star-forming galaxies and incorporating closely interacting pairs and ongoing mergers. These galaxies have very high star formation rates (SFRs) per unit surface, hence all of them are rather radio-bright. In what follows we shall briefly introduce the galaxies and their pertinent properties, subdividing them into several categories. All subsequent tables of this paper follow this categorisation using dashed horizontal lines.

Dwarf galaxies: there are five low-mass galaxies in our sample. II Zw 40 and II Zw 70 are classical blue compact dwarf galaxies (BCGDs). BCDGs are also called H II galaxies because they are dominated by giant $\mathrm{H}$ II regions occupying much of their total volumes. Their emission-line spectra indicate that they are metal-poor, while they are gas-rich and are experiencing intense bursts of star formation. II $\mathrm{Zw} 40$ was observed in the radio continuum by Klein et al. (1984b), Klein et al. (1991), and by Deeg et al. (1993). II Zw 70 is a small and distant BCDG meaning that existing radio continuum measurements (Klein et al. 1984b; Skillman \& Klein 1988; Deeg et al. 1993) only provide integrated flux densities. The high-frequency radiation of these two galaxies is purely thermal.

IC 10, NGC 1569, and NGC 4449 are nearby starbursting dwarf galaxies. They have properties that are rather similar to those of BCDGs. They are gas-rich, too, but their metallicities are not so low.

Because of its proximity to the Galactic plane, IC 10 is a tricky case for radio continuum observations. In particular at low frequencies, at which interferometric observations are indispensable, imaging suffers from contamination by spurious sidelobes from radio continuum structures in the Galactic plane. Another worry is thermal absorption through the plane at the lowest frequencies. Useful measurements for our purpose have been carried out by Klein et al. (1983); Klein \& Gräve (1986); Chyży et al. (2003), and by Chyży et al. (2016).

NGC 1569 is considered as a template for a low-mass galaxy with an evolved starburst. Israel \& de Bruyn (1988) were the first to notice that the synchrotron spectrum is not a simple power-law, but "has a high-frequency cutoff at $8 \pm 1 \mathrm{GHz}$ ". This rapid decline is not obvious in the total radio spectrum, due to a high fraction of thermal radio emission, which was derived by Israel \& de Bruyn (1988) from the $\mathrm{H} \alpha$ emission. Numerous radio continuum studies at a multitude of frequencies, which aimed at an understanding of cosmic-ray propagation into the halo regime of this dwarf galaxy (Klein \& Gräve 1986; Lisenfeld et al. 2004; Kepley et al. 2010; Purkayastha 2014), prove its intense star formation. It is among the radio-brightest in our sample and possesses a low-frequency radio halo.

Another such nearby template of a starburst dwarf galaxy, NGC 4449, has been observed in the radio continuum by Klein \& Gräve (1986, $24.5 \mathrm{GHz})$, Chyży et al. (2000, 4.9, $8.5 \mathrm{GHz})$, Chyży et al. (2011, 2.7, 4.9 GHz), Srivastava et al. (2014, 150, 325, $610 \mathrm{MHz}$ ), and by Purkayastha (2014, $350 \mathrm{MHz}$ ). This galaxy is also characterised by a high SFR, which was most likely triggered by the close passage of another - yet-lower-mass - galaxy. NGC 4449 also possesses a low-frequency radio halo.

Interacting galaxies: upon close inspection, almost all galaxies are gravitationally interacting with another to some extent. In the present sample, NGC 4490/85, NGC 5194/95 (M 51), and NGC 4631 (with nearby dwarf elliptical NGC 4627 and the more massive disk galaxy NGC 4652) have nearby and obviously interacting companion galaxies. These interactions are the likely cause of the intense ongoing star formation in these galaxies, with the close companions stirring up the gas and giving rise to gas compression and subsequent star formation out of the molecular gas.

Radio continuum studies of NGC 4490/85, a closely interacting pair of galaxies, were reported by Viallefond et al. (1980, 
$1.4 \mathrm{GHz})$, Clemens et al. (1999, 1.49, 4.86, 8.44, $15.2 \mathrm{GHz})$, and by Klein et al. (1983, 24.5 GHz). Nikiel-Wroczyński et al. (2016) published a multi-frequency radio continuum study of this system using archival VLA data and new GMRT observations at $610 \mathrm{MHz}$. The highest frequency at which NGC 4490/85 has been studied is $25 \mathrm{GHz}$ (Klein 1983).

M 51 has been the target of numerous radio continuum studies, which in the early days aimed at measuring the total radio continuum spectrum and studying cosmic-ray propagation (Klein et al. 1984a), and later focused on investigating the structure of the large-scale magnetic field located within it (e.g. Neininger 1992; Fletcher et al. 2011; Mao et al. 2015). The rich set of flux-density measurements available for this galaxy required a careful selection, with only the most reliable data in terms of confusion and missing short spacings being retained. Klein et al. (1984a) inferred a simple power-law for the synchrotron spectrum of M 51 .

After the discovery of an extended radio continuum halo at 610 and $1412 \mathrm{MHz}$ around NGC 4631 by Ekers \& Sancisi (1977) there has been a large number of observations at higher frequencies (Hummel \& Dettmar 1990; Hummel et al. 1991; Golla 1999; Irwin et al. 2012). These mostly aimed at studying cosmic-ray transport out of the disk and at investigating the morphology of the magnetic field of this galaxy. The high SFR is probably caused by the gravitational interaction with the neighbouring galaxies NGC 4627 and NGC 4652, rendering this galaxy a rather radio-bright one.

Merging galaxies: in the case of ongoing mergers, star formation is extreme, owing to the collision of molecular clouds in the merging centres of the two galaxies.

NGC 4038/39, the "Antenna Galaxy", represents one of the classical nearby ongoing mergers of two galaxies. Chyży \& Beck (2004) and Basu et al. (2017) performed thorough radio continuum studies, with the emphasis on the linear polarisation and on the resulting morphology of the large-scale magnetic field in NGC 4038/39. In separating the thermal from the nonthermal radio emission, they inferred a simple power-law for the total synchrotron spectrum of this system.

NGC 6052, which became known as a so-called "clumpy irregular galaxy" under its label Mkn 297 (Heidmann 1979), is revealed as a close merger of two galaxies oriented perpendicularly by HST images (Holtzman et al. 1996). It has a high radio brightness. High-resolution radio continuum observations of this system were reported by Deeg et al. (1993).

Starburst galaxies: NGC 2146 and NGC 3034 (M 82) are classical nearby starburst galaxies, again with the intense starforming activity resulting from gravitational interaction with a nearby galaxy or from a past merger event. A comprehensive radio continuum study of NGC 2146 was reported by Lisenfeld et al. (1996), who investigated the cosmic-ray transport in this galaxy. NGC 3034 (M82) has been the target of a large number of radio continuum studies across a large frequency range. Adebahr et al. (2013) presented observations at $\lambda \lambda 3,6$, 22 , and $92 \mathrm{~cm}$, using the VLA and the WSRT. Varenius et al. (2015) made dedicated measurements with LOFAR to image the radio continuum of M 82 at 118 and $154 \mathrm{MHz}$. However, such low-frequency measurements are not relevant for our present study, since at frequencies below about $1 \mathrm{GHz}$ various effects may shape the synchrotron spectra in such a way as to produce deviations from a simple power-law (see Sect. 3.2). Klein et al. (1988) published observations of M 82 at $32 \mathrm{GHz}$, the highest frequency at which thermal radiation from dust is still insignificant.

NGC 3079 and NGC 3310 are starburst galaxies which can be considered as transition phases towards AGN (active galactic nucleus) activity. NGC 3079 exhibits a focused wind emerging from its centre and giving rise to a "figure- 8 " bow-shock structure seen in the nonthermal radio continuum (Duric et al. 1983; Duric \& Seaquist 1988). NGC 3310 has recently swallowed one of its dwarf companion galaxies (see, e.g. Kregel \& Sancisi 2001), this having led to the enormous starburst. High-resolution radio continuum observations were performed by Duric et al. (1986), with the aim to study the distribution of cosmic rays in this galaxy.

\section{The data}

\section{1. $24.5 \mathrm{GHz}$ measurements}

The observations at $24.5 \mathrm{GHz}$ had been performed in perfect weather during test measurements in February 1982. The $K$-band maser receiver system with a bandwidth $100 \mathrm{MHz}$ had been installed in the primary focus of the Effelsberg 100-m telescope. The half power beam width (HPBW) was measured to be $38^{\prime \prime} \pm 1^{\prime \prime}$. The frontend was equipped with two horns, giving an angular beam separation of $114^{\prime \prime} \pm 2$ " on the sky. The aperture efficiency of the 100-m telescope was $19 \%$ at the time of the observations. The pointing and focus of the telescope were checked using the point source 3C 286, with small maps centred on this source providing also the flux calibration. The flux density scale is that of Baars et al. (1977). For each galaxy, six coverages were taken between $50^{\circ}$ and $80^{\circ}$ elevation (corresponding to system temperatures of 79 and $49 \mathrm{~K}$, respectively, on the sky), using a scan separation of $10^{\prime \prime}$. These were stacked to yield final maps with a root mean square (rms) noise of $2.9 \mathrm{mJy} /$ beam area. The observing procedure and reduction technique is essentially the same as described by Emerson et al. (1979). The sample selection was governed by the radio brightness and sizes of the target galaxies, dictated by the rather high frequency and the comparatively small bandwidth of the maser receiver. Since those were the first (and only) measurements of this kind, the authors attempted to include star-forming galaxies with a variety of properties (low- and high-mass galaxies, and interacting systems).

\subsection{Literature search}

In what follows, we describe the compilation of the data used below 24.5 GHz. All published flux densities used here were checked for the calibration scale and, wherever necessary, were scaled to the common scale of Baars et al. (1977). At the highest frequencies $(24.5$ and $32 \mathrm{GHz}$ ), the Baars scale is $\sim 1 \%$ higher than that properly extended to higher frequencies by Perley \& Butler (2013). This small diifference does not affect our analysis.

A careful selection was made to ensure that the most reliable data would be used. We discarded interferometric measurements that might underestimate the flux density as a result of missing short spacings (mostly relevant above about $1.4 \mathrm{GHz}$ ), but also single-dish measurements that might overestimate the flux density owing to source confusion (mostly relevant below about $5 \mathrm{GHz}$ ). If several measurements were available at the same or a neighbouring frequency, we calculated the error-weighted average at the corresponding mean frequency. The resulting data compilation is presented in tabular form in Appendix B. 
For each galaxy, the tables give the mean frequency in $\mathrm{GHz}$ (Col. 1), the flux density (Col.2) and its error (Col.3) in mJy, and the reference to the flux density measurement (Col.4). In a few cases, we have determined flux densities at $325 \mathrm{MHz}$ and 1.4 GHz using the Westerbork Northern Sky Survey (WENSS; Rengelink et al. 1997) and the NRAO VLA Sky Survey (NVSS; Condon et al. 1998), respectively. These are galaxies with small angular sizes meaning that flux loss by interferometric measurements is not an issue. Finally, some flux densities were recently obtained in the course of other observing programmes (VLA, Effelsberg).

We decided to utilise only flux densities above $0.3 \mathrm{GHz}$, since at lower frequencies a number of astrophysical effects such as thermal absorption may shape the synchrotron spectra in such a way as to produce deviations from a simple power-law. It would require more free parameters, rendering the fit results less reliable when taking these processes into account. The synchrotron spectra obtained here may serve though to extrapolate them to lower frequencies and thus provide a firm "leverage" for low-frequency studies.

\subsection{Ancillary data}

Apart from the radio data, we collected a set of ancillary data from the literature for the galaxies that allowed us to quantify their SFR, H $\alpha$ flux, stellar mass, metallicity, and optical sizes. The data, together with the distances, are listed in Table 1.

As a measure of the stellar mass we used the infrared $K_{\mathrm{S}}$ luminosity, $L_{\mathrm{K}}$, which we calculated from the total (extrapolated) $K_{\mathrm{S}}$ flux, $f_{\mathrm{K}}$, as $L_{\mathrm{K}}=v f_{\mathrm{K}}(v) 4 \pi D^{2}$ (where $D$ is the distance and $v$ is the frequency of the $K$-band $\left.{ }^{1}, 1.38 \times 10^{14} \mathrm{~Hz}\right)$. The fluxes in the $K_{\mathrm{S}}(2.17 \mu \mathrm{m})$ band were taken from the 2MASS Extended Source Catalog (Jarrett et al. 2000), the 2MASS Large Galaxy Atlas (Jarrett et al. 2003), and from the 2Mass Extended Objects Final Release.

We calculated the SFR as a combination of $24 \mu \mathrm{m}$ and $\mathrm{H} \alpha$ fluxes, following Kennicutt et al. (2009):

$S F R=5.5 \times 10^{-42} \frac{L(\mathrm{H} \alpha)+L(24 \mu \mathrm{m})}{\operatorname{erg~s}^{-1}} M_{\odot} \mathrm{yr}^{-1}$.

For the $24 \mu \mathrm{m}$ flux, we used, whenever possible, the flux measured with MIPS on the Spitzer satellite. Only in those cases for which no Spitzer data were available (IC 10 and NGC 4038), were data from the IRAS satellite at $25 \mu \mathrm{m}$ used, neglecting the small central wavelength difference. By combining mid-infrared and $\mathrm{H} \alpha$ fluxes we take into account both unobscured and dustenshrouded star formation and circumvent the uncertain extinction correction of the $\mathrm{H} \alpha$ flux.

\section{Spectral fitting}

\subsection{Radio spectra}

Following Eq. (1) we fitted the radio data with the sum of thermal and nonthermal radio emission.

In order to predict the synchrotron emission of a model galaxy, the first step is to calculate the relativistic electron particle density, $N(E)$, that is, the number density of relativistic electrons at energy $E$ within the energy interval $\mathrm{d} E$. The synchrotron

\footnotetext{
1 We note that - unfortunately - there exists definitions for the $K$-band in both, optical and radio astronomy, both of which have to be used in the present paper and must not be confused.
}

emission can then be calculated by convolving this distribution with the synchrotron emission of a single electron:

$S_{\text {nth }}(v) \propto \int_{1}^{\infty}\left(\frac{v}{v_{\mathrm{c}}}\right)^{0.3} \mathrm{e}^{-v / v_{\mathrm{c}}} N(\gamma) \mathrm{d} \gamma$,

where $\gamma=E /\left(m_{\mathrm{e}} c^{2}\right)$ is the Lorentz factor, $m_{\mathrm{e}}$ the electron rest mass and

$v_{\mathrm{c}}=\frac{3}{4 \pi} \frac{e B_{\perp}}{m_{\mathrm{e}} c} \gamma^{2}$

which is the critical frequency, $e$ is the electron charge, and $B_{\perp}$ the strength of the magnetic field component perpendicular to the direction of the relativistic electron's velocity. The critical frequency is close to the peak of the electron spectrum and represents, within a factor of a few, the frequency where the relativistic electrons emit most of their energy (see, e.g. Klein \& Fletcher 2015). The shape of the synchrotron spectrum emitted by a single electron is such that for frequencies above $v_{\mathrm{c}}$ the emission decreases exponentially, whereas for lower frequencies there is power-law, $v^{0.3}$.

If the electron energy distribution is a power-law, $N(E) \propto E^{-g}$, we can calculate the synchrotron emission to a good accuracy by assuming that an electron with $E$ emits the entire synchrotron radiation at the critical frequency (see Klein \& Fletcher 2015),

$S_{\mathrm{nth}}(v)=\left.N\left[E\left(v_{\mathrm{c}}\right)\right] \frac{\mathrm{d} E}{\mathrm{~d} t}\right|_{\mathrm{syn}} \cdot \frac{\mathrm{d} E}{\mathrm{~d} v} \propto B_{\perp}^{\frac{g+1}{2}} v^{-\frac{g-1}{2}}$.

In the following we explain the four different models that we considered for the synchrotron spectrum. We chose these models with the goal to cover the range from a spectrum with constant slope to a maximally curved synchrotron spectrum within simplified yet realistic scenarios.

Spectrum with a constant slope: in the first case we adopt a synchrotron spectrum with a constant slope over the entire frequency range,

$S_{\mathrm{nth}}(v)=S_{\mathrm{nth}, 0}\left(\frac{v}{v_{0}}\right)^{-\alpha_{\mathrm{nth}}}$,

where we take $v_{0}=1 \mathrm{GHz}$ in this paper. For the other three cases, we consider simple models, spatially integrated over the entire galaxy, which allows us to predict the expected synchrotron emission in different scenarios.

Spectrum curved due to energy losses: here, we consider a steady-state, closed-box model. Since we are only interested in the total $N(E)$, integrated over the entire galaxy, we do not need to take into account propagation of the relativistic particles, as long as it is energy-independent. In this approximation, the corresponding equation for the relativistic electron particle density is:

$\frac{\partial}{\partial E}[b(E) N(E)]=\left(\frac{E}{m_{\mathrm{e}} c^{2}}\right)^{-g_{\mathrm{inj}}} q_{\mathrm{SN}} v_{\mathrm{SN}}$.

This equation takes into account acceleration in supernova remnants (SNRs) with a source spectrum as $\gamma^{-g_{\text {inj }}} q_{\mathrm{SN}} v_{\mathrm{SN}}$ (where $v_{\mathrm{SN}}$ is the supernova rate, $q_{\mathrm{SN}}$ is the number of relativistic electrons produced per supernova and per unit energy interval, and $g_{\text {inj }}$ is 
Table 1. Ancillary data for the galaxy sample.

\begin{tabular}{|c|c|c|c|c|c|c|c|c|c|}
\hline Galaxy & $\begin{array}{c}D \\
{[\mathrm{Mpc}]}\end{array}$ & $\begin{array}{l}d^{1} \\
{[\mathrm{kpc}]}\end{array}$ & $12+\log (\mathrm{O} / \mathrm{H})$ & Ref. $^{2}$ & $\begin{array}{c}\log \left(L_{\mathrm{K}}\right)^{3} \\
{\left[L_{\odot, k}\right]}\end{array}$ & $\begin{array}{c}\log \left(L_{24} \mu \mathrm{m}\right)^{4} \\
{\left[\mathrm{erg} \mathrm{s}^{-1}\right]}\end{array}$ & $\begin{array}{c}F(\mathrm{H} \alpha)^{5} \\
{\left[\mathrm{erg} \mathrm{s}^{-1} \mathrm{~cm}^{-2} \text { ] }\right.}\end{array}$ & Ref. $^{6}$ & $\begin{array}{c}\log (S F R)^{7} \\
{\left[M_{\odot} \mathrm{yr}^{-1}\right]}\end{array}$ \\
\hline IC 10 & 0.9 & 1.6 & 8.30 & 1 & 8.79 & 40.61 & -9.63 & 2 & -0.93 \\
\hline II Zw 40 & 10.3 & 1.3 & 8.12 & 2 & 8.42 & 42.41 & -10.81 & 1 & 0.02 \\
\hline II Zw 70 & 23.1 & 4.8 & 7.86 & 3 & 8.94 & 41.83 & -12.08 & 1 & -0.56 \\
\hline NGC 1569 & 2.9 & 3.3 & 8.16 & 4 & 9.05 & 42.03 & -10.05 & 2 & -0.21 \\
\hline NGC 4449 & 3.7 & 5.0 & 8.23 & 5 & 9.57 & 41.83 & -10.64 & 2 & -0.55 \\
\hline NGC 4490 & 8.1 & 15.8 & 8.39 & 9 & 10.21 & 42.63 & -10.86 & 2 & 0.03 \\
\hline NGC 4631 & 6.3 & 26.4 & 8.75 & 6 & 10.35 & 42.69 & -10.70 & 2 & 0.02 \\
\hline NGC 5194 & 7.6 & 30.2 & 8.86 & 6 & 10.90 & 43.03 & -10.79 & 2 & 0.26 \\
\hline NGC 4038 & 21.3 & 33.4 & 8.74 & 8 & 11.12 & 43.65 & -10.93 & 3 & 2.01 \\
\hline NGC 6052 & 70.4 & 16.8 & 8.85 & 10 & 10.76 & 43.74 & -11.66 & 3 & 1.04 \\
\hline NGC 2146 & 22.4 & 34.5 & 8.68 & 5 & 11.21 & 44.11 & -11.18 & 3 & 1.20 \\
\hline NGC 3034 & 3.8 & 12.1 & 9.12 & 6 & 10.63 & 43.85 & -10.11 & 2 & 0.93 \\
\hline NGC 3079 & 19.1 & 45.3 & 8.89 & 7 & 10.99 & 43.22 & -11.42 & 3 & 0.35 \\
\hline NGC 3310 & 18.1 & 9.4 & 8.75 & 7 & 10.40 & 43.39 & -10.95 & 3 & 0.64 \\
\hline
\end{tabular}

Notes. ${ }^{(1)}$ Optical diameter in kpc, calculated from $d_{25}$ in LEDA. ${ }^{(2)}$ References for the oxygen abundance: (1) Magrini \& Gonçalves (2009); (2) Thuan \& Izotov (2005); (3) Kehrig et al. (2008); (4) Kobulnicky \& Skillman (1997); (5) Engelbracht et al. (2008); (6) Moustakas et al. (2010); (7) Robertson et al. (2013); (8) Bastian et al. (2009); (9) Pilyugin \& Thuan (2007); (10) Sage et al. (1993). ${ }^{(3)}$ Decimal logarithm of the luminosity in the $K$-band in units of the solar luminosity in the $K_{\mathrm{S}}$-band $\left(L_{K, \odot}=5.0735 \times 10^{32} \mathrm{erg} \mathrm{s}^{-1}\right) .{ }^{(4)}$ Decimal logarithm of the luminosity at $24 \mu \mathrm{m}$, derived from Spitzer MIPS fluxes as $L_{24} \mu \mathrm{m}=v f_{24} \mu \mathrm{m} \times 4 \pi D^{2}$. The fluxes were obtained from Engelbracht et al. (2008, II Zw 40, NGC 1569, NGC 2146, NGC 3310, NGC 4449, NGC 3079), Dale et al. (2009, NGC 3034, NGC 4490, NGC 4631, NGC 5194), Brown et al. (2014, NGC 6052), or directly from the Spitzer archive (https://irsa.ipac. caltech. edu) (II Zw 70). For two galaxies (IC 10 and NGC 4038), no Spitzer MIPS data were available and we used IRAS $25 \mu \mathrm{m}$ instead, neglecting the slight difference in the central wavelength. ${ }^{(5)}$ Decimal logarithm of the $\mathrm{H} \alpha$ flux, corrected for N II contribution and for Galactic foreground extinction. ${ }^{(6)}$ References for the H $\alpha$ fluxes: (1) Gil de Paz et al. (2003); (2) Kennicutt et al. (2008); (3) Moustakas \& Kennicutt (2006). ${ }^{(7)}$ Decimal logarithm of the SFR, calculated with Eq. (2).

the injection spectral index), and the radiative energy losses of the relativistic electrons $\mathrm{d} E / \mathrm{d} t=b(E)$. The injection spectral index, $g_{\text {inj }}$ is predicted by shock acceleration theory to be 2.1 (Drury et al. 1994; Berezhko \& Völk 1997). Equation (7) can be solved by integration and gives

$N(E)=\left(\frac{E}{m_{\mathrm{e}} c^{2}}\right)^{-g_{\mathrm{inj}}+1} \frac{q_{\mathrm{SN}} v_{\mathrm{SN}} m_{\mathrm{e}} c^{2}}{b(E)\left(g_{\mathrm{inj}}-1\right)}$.

Thus, the spectral shape of $N(E)$ and of the resulting synchrotron emission depend on the energy dependence of the energy losses. The most relevant energy losses for $\mathrm{CR}$ electrons in the $\mathrm{GHz}$ range are inverse-Compton and synchrotron losses,

$b(E)_{\mathrm{syn}+i \mathrm{C}}=\left.\frac{\mathrm{d} E}{\mathrm{~d} t}\right|_{\mathrm{syn}+i \mathrm{C}}=C_{\mathrm{syn}+i \mathrm{C}} E^{2}$,

with $C_{\text {syn }+i \mathrm{C}} \propto\left(U_{\text {rad }}+U_{\mathrm{B}}\right), U_{\text {rad }}$ being the energy density of the radiation field below the Klein-Nishina limit, and $U_{\mathrm{B}}$ the energy density of the magnetic field. We furthermore consider bremsstrahlung and adiabatic losses, which depend linearly on $E$ and can thus become relevant at lower energies/frequencies.

$b(E)_{\mathrm{ad}+\text { brems }}=\left.\frac{\mathrm{d} E}{\mathrm{~d} t}\right|_{\mathrm{ad}+\text { brems }}=C_{\mathrm{ad}+\text { brems }} E$.

Here $C_{\text {ad+brems }}$ is a constant that depends on the gas density and advection velocity gradient. Taking both kinds of energy loss into account results in an energy distribution of the relativistic electrons that exhibits a (very shallow) change of slope centered at the break energy, $E_{\mathrm{b}}=C_{\mathrm{ad}+\text { brems }} / C_{\mathrm{syn}+i \mathrm{C}}$,

$N(E) \propto \frac{E^{-g_{\text {inj }}}}{E / E_{\mathrm{b}}+1}$.
The corresponding curved synchrotron spectrum reads

$S_{\mathrm{nth}}(v)=S_{\mathrm{nth}, 0} \frac{\left(\frac{v}{v_{0}}\right)^{-\alpha_{\mathrm{nth}}}}{\left(\frac{v}{v_{\mathrm{b}}}\right)^{0.5}+1}$

where $v_{\mathrm{b}}=v\left(\gamma_{\mathrm{b}}\right)$ is the break frequency at which the change of slope takes place, and $\alpha_{\text {nth }}=\frac{g_{\text {inj }}-1}{2}$ is the low-frequency synchrotron spectral index.

Spectrum with a break: we can produce a much more pronounced break in an open-box model, in which the relativistic electrons can escape from a finite halo with vertical extent $z_{\text {halo }}$ (see Lisenfeld et al. 2004, for a detailed discussion). The sharpest break occurs when the relativistic electrons propagate by convection because the scalelength (i.e. the distance over which relativistic electrons can propagate before losing their energy) of diffusion, being a stochastic process, has a much weaker dependence on energy and therefore produces a shallower break. We only take into account inverse-Compton and synchrotron losses, according to Eq. (9). The propagation equation for the relativistic electrons accelerated in SNRs in the galactic plane at $z=0$ and moving with constant convection speed $V_{\mathrm{c}}$ in the $\mathrm{z}$-direction perpendicular to the disk is in this case

$\frac{\partial N(E, z)}{\partial z} V_{\mathrm{c}}-\frac{\partial}{\partial E}\left[C_{\mathrm{syn}+i \mathrm{C}} E^{2} N(E, z)\right]=\delta(z)\left(\frac{E}{m_{\mathrm{e}} c^{2}}\right)^{-g_{\mathrm{inj}}} q_{\mathrm{SN}} v_{\mathrm{SN}}$,

with $\delta(z)$ being the one-dimensional $\delta$-function. The solution of this equation is given by

$N(E, z)=\left\{\begin{array}{ll}\frac{q_{\mathrm{SN}} v_{\mathrm{SN}}}{2 V_{\mathrm{c}}}\left(\frac{E}{m_{\mathrm{e}} c^{2}}\right)^{-g_{\mathrm{inj}}}\left(1-\frac{z}{z_{\max }}\right)^{g_{\mathrm{inj}}-2} & z<z_{\max } \\ 0 & z \geq z_{\max }\end{array}\right.$, 
Table 2. Shapes of the radio (synchrotron and free-free) emission considered in the fitting process.

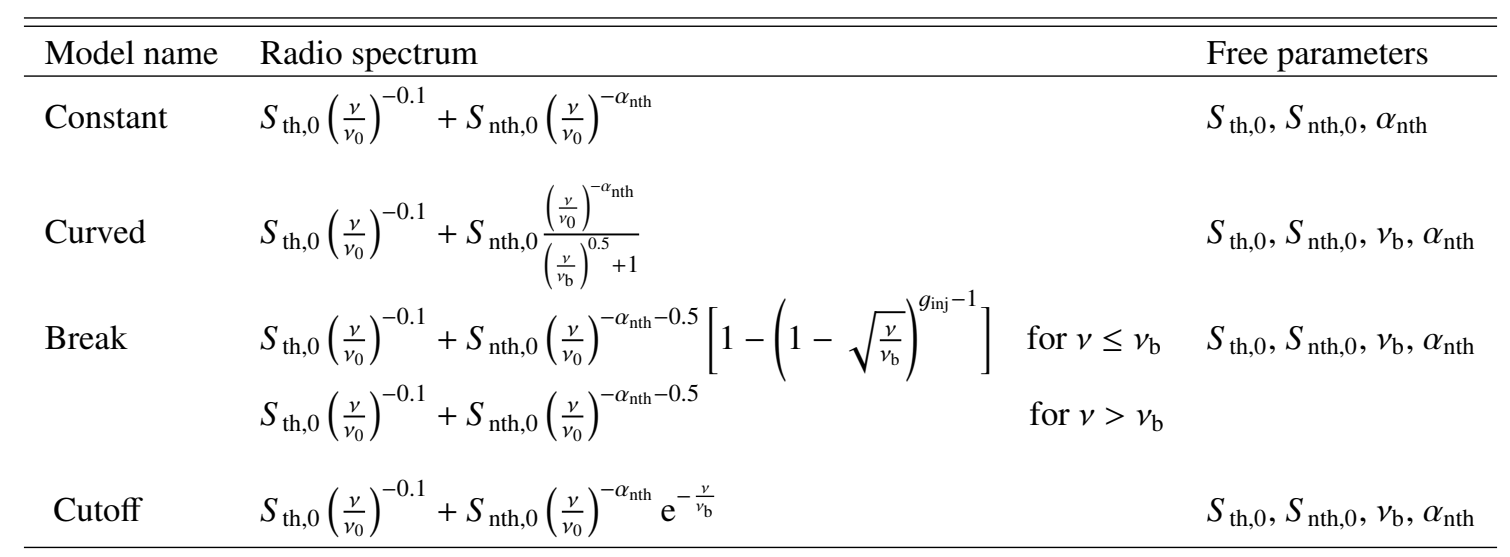

Notes. We take $v_{0}=1 \mathrm{GHz}$ in this paper.

where $z_{\max }=V_{\mathrm{c}} /\left(E C_{\mathrm{syn}+i \mathrm{C}}\right)$ is the maximum distance that a relativistic electron can travel before having lost its energy to below $E$. The total relativistic electron density in the galaxy is calculated by integrating Eq. (14) from $z=0$ to $z=\min \left(z_{\max }, z_{\text {halo }}\right)$. We then assume that the relativistic electrons emit all their energy at $v_{\mathrm{c}}$ and obtain for the synchrotron emission,

$S_{\mathrm{nth}}(v)=\left\{\begin{array}{ll}S_{\mathrm{nth}, 0}\left(\frac{v}{v_{0}}\right)^{-\alpha_{\mathrm{nth}}-0.5}\left[1-\left(1-\sqrt{\frac{\nu}{v_{\mathrm{b}}}}\right)^{g_{\mathrm{inj}}-1}\right] & v \leq v_{\mathrm{b}} \\ S_{\mathrm{nth}, 0}\left(\frac{v}{v_{0}}\right)^{-\alpha_{\mathrm{nth}}-0.5} & v>v_{\mathrm{b}}\end{array}\right.$.

The resulting spectrum has a break at $v_{\mathrm{b}}=v_{\mathrm{c}}\left(E_{\mathrm{b}}\right)$, with break energy $E_{\mathrm{b}}=V_{\mathrm{c}} /\left(z_{\text {halo }} C_{\mathrm{syn}+i \mathrm{C}}\right)$. The spectral index changes from $\alpha_{\text {nth }}+0.5$ at $v>v_{\mathrm{b}}$ to $\alpha_{\text {nth }}$ for $v \ll v_{\mathrm{b}}$, that is, it changes by 0.5 . The break produced is much more pronounced than the shallow change of spectral index due to different energy losses (this can be clearly seen in the figures in Appendix $C$ where the synchrotron spectra of the different models are shown and can be compared).

Spectrum with an exponential cutoff: finally, we consider the case that the distribution of relativistic electrons ends abruptly at a certain Lorentz factor $\gamma_{\max }$. Assuming continuous pitch-angles, thus following the model of Jaffe \& Perola (1973), an exponential cutoff is produced in the synchrotron spectrum according to Eq. (5) whenever there is an abrupt cutoff in the energy spectrum of the relativistic electrons (Jaffe \& Perola 1973; Kardashev 1962). A cutoff in energy can occur in a single-injection scenario because the highest-energy electrons lose their energy much faster than lower-energy electrons, meaning that their population becomes completely depopulated after a characteristic energyloss time. The location of the exponential cutoff in the radiation spectrum depends on the relative time scales for acceleration, energy losses, and escape of the particles (Schlickeiser 1984). The synchrotron spectrum in this case is

$S_{\text {nth }}(v)=S_{\text {nth }, 0}\left(\frac{v}{v_{0}}\right)^{-\alpha_{\text {nth }}} \mathrm{e}^{-\frac{v}{v_{\mathrm{b}}}}$.

Table 2 summarises the four models, together with the free parameters determined in the spectral fitting.

\subsection{Fitting method}

For each galaxy, we fit four models: constant, curved, break, and cutoff. The shapes of the model radio spectra are presented in the previous subsection and recapitulated in Table 2, along with the list of free parameters. The only fixed parameter was the slope of the (optically thin) thermal emission, that is, $\alpha_{\text {th }}=0.1$. We require $S_{\text {th }}$ to be positive for all four models. In addition, we provide the following bounds for some parameters: $S_{\text {nth }} \geq 0$ and $1 \leq v_{\mathrm{b}} \leq 50 \mathrm{GHz}$ for the break model and $v_{\mathrm{b}} \geq 0$ for the cutoff model.

For each model, the best fit is obtained via the Trust Region Reflective ("trf") algorithm for optimisation, well suited to efficiently explore the whole space of variables for a bound-constrained minimisation problem (Branch et al. 1999). In Appendix C, we provide more detailed information and show the best fits of all four models (constant, curved, break, and cutoff) for an easier comparison, together with a table of their bestfit parameters.

\section{Results}

Figure 1 shows the best fits out of the four tested cases (constant, curved, break, or cutoff, solid red line). In these plots, we also display the free-free (thermal, dotted cyan line) and synchrotron (nonthermal, dashed green line) components of the models. The observed radio continuum flux densities minus the fitted nonthermal component is represented by cyan stars, while the observed minus the fitted thermal component is depicted by green crosses. The optimal values of the parameters are listed in the lower left part of the figure, and their errors are given in terms of the standard deviation of 1000 generated models (for more detailed information see Appendix C).

In Table 3 we compile the fit results obtained from the bestfit model, with the total flux density at $1 \mathrm{GHz}$ and its error listed in Col. 2, the fraction of thermal emission at $1 \mathrm{GHz}$ and its error in Col.3, the nonthermal spectral index and its error in Col. 4, the break frequency in $\mathrm{GHz}$ and its error in Col.5, the reduced $\chi_{v}^{2}$ in Col. 6, and the name of the best-fit model in Col. 7.

In general, the best fit was selected as the lowest reduced chi-squared, $\chi_{v}^{2}$. Apart from this, the resulting thermal emission was an asset of whether the fits are physically meaningful. Thus, we rejected fits in spite of an acceptable $\chi_{v}^{2}$ if the fitted thermal radio emission was unphysically low. In addition, an important test was to look at the resulting thermal flux densities obtained at each frequency after subtracting the computed nonthermal flux density from the total (observed). A fit only makes sense if the resulting thermal flux densities so obtained obey to the expected optically thin spectrum with slope -0.1 . This is shown by the 
U. Klein et al.: Synchrotron spectra of galaxies
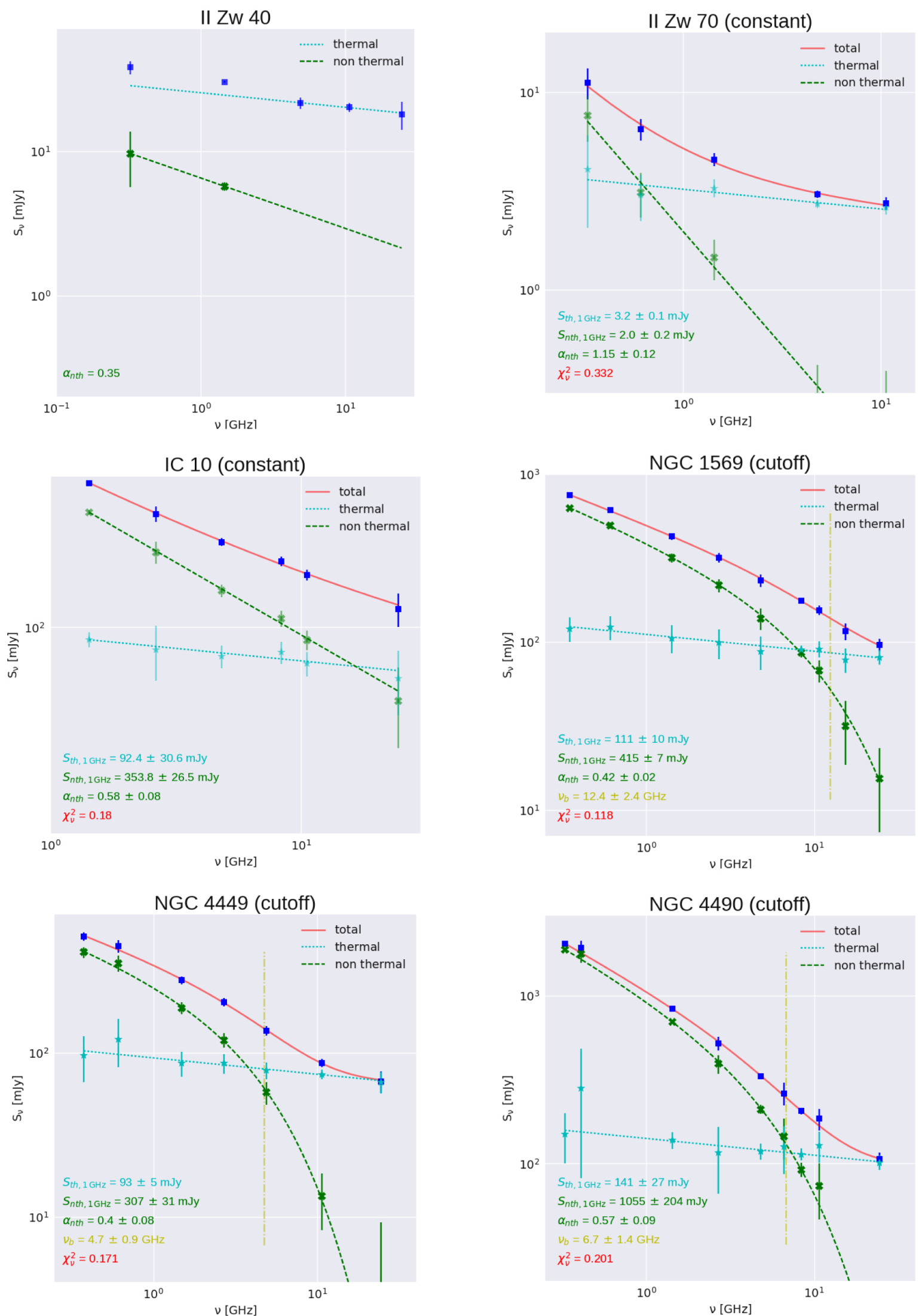

Fig. 1. Radio continuum spectra of the sample galaxies. Except for II Zw 40 (see text for the procedure and plotted lines here), the plots show the following: measured flux densities (blue squares), best fit of the radio continuum spectrum (solid red line). The best parameters are listed in the lower left part of the figure along with the reduced chi-square $\left(\chi_{v}^{2}\right)$. The free-free (thermal) and synchrotron (nonthermal) components of the models are depicted by the dotted cyan line and dashed green line, respectively. The green crosses represent the observed nonthermal component (i.e. the modelled thermal component removed from the observational data). The cyan stars delineate the observed thermal component (i.e. the modelled nonthermal component subtracted from the observational data). The vertical yellow dash-dotted line marks the break frequency $v_{\mathrm{b}}$. (To be continued in Fig. A.1.) 
Table 3. Fit results.

\begin{tabular}{|c|c|c|c|c|c|c|}
\hline Galaxy & $\begin{array}{r}S_{\text {tot, } 1 \mathrm{GHz}} \\
{[\mathrm{mJy}]}\end{array}$ & $f_{\text {th, } 1 \mathrm{GHz}}$ & $\alpha_{\text {nth }}$ & $\begin{array}{c}v_{\mathrm{b}} \\
{[\mathrm{GHz}]}\end{array}$ & $\chi_{v}^{2}$ & Best fit \\
\hline II Zw 40 & 32 & 0.80 & 0.35 & - & - & - \\
\hline II Zw 70 & $5.2 \pm 0.2$ & $0.62 \pm 0.03$ & $1.15 \pm 0.12$ & - & 0.33 & constant \\
\hline IC 10 & $446 \pm 40$ & $0.21 \pm 0.07$ & $0.58 \pm 0.08$ & - & 0.18 & constant \\
\hline NGC 1569 & $494 \pm 12$ & $0.22 \pm 0.02$ & $0.42 \pm 0.02$ & $12.4 \pm 2.4$ & 0.12 & cutoff \\
\hline NGC 4449 & $342 \pm 32$ & $0.27 \pm 0.03$ & $\underline{0.40 \pm 0.08}$ & $4.7 \pm 0.9$ & 0.17 & cutoff \\
\hline NGC 4490 & $1051 \pm 206$ & $0.13 \pm 0.04$ & $0.57 \pm 0.09$ & $6.8 \pm 1.4$ & 0.20 & cutoff \\
\hline NGC 4631 & $1637 \pm 75$ & $0.14 \pm 0.01$ & $0.57 \pm 0.03$ & $6.6 \pm 0.4$ & 2.08 & cutoff \\
\hline NGC 5194 & $1788 \pm 464$ & $0.11 \pm 0.03$ & $0.67 \pm 0.10$ & $7.2 \pm 0.6$ & 0.62 & cutoff \\
\hline NGC 4038 & $683 \pm 160$ & $0.13 \pm 0.04$ & $0.71 \pm 0.09$ & $8.2 \pm 3.8$ & 0.01 & break \\
\hline NGC 6052 & $129 \pm 10$ & $0.08 \pm 0.01$ & $0.56 \pm 0.04$ & $2.4 \pm 0.5$ & 0.32 & break \\
\hline$\overline{\mathrm{NGC}} 21 \overline{4} \overline{6}$ & $1359 \pm 18$ & $0.16 \pm 0.01$ & $0 . \overline{5} 1 \pm 0.02$ & $6.2 \pm 0.5$ & $0 . \overline{4}$ & cutoff \\
\hline NGC 3034 & $9043 \pm 175$ & $0.14 \pm 0.01$ & $0.40 \pm 0.02$ & $11.2 \pm 1.0$ & 0.45 & cutoff \\
\hline NGC 3079 & $1111 \pm 104$ & $0.10 \pm 0.02$ & $0.74 \pm 0.01$ & $9.0 \pm 2.0$ & 1.59 & break \\
\hline NGC 3310 & $470 \pm 16$ & $0.19 \pm 0.01$ & $0.60 \pm 0.03$ & $1.2 \pm 0.3$ & 0.40 & break \\
\hline
\end{tabular}

blue data points in each diagram, with the errors taken from those of the measured total flux densities.

The most striking result of our fits is that most objects need strongly curved (break or cutoff) synchrotron spectra in order to satisfactorily fit the total radio spectrum. Apparent exceptions are the three dwarf galaxies IC 10, II Zw 40, and II Zw 70 . IC 10 and II Zw 70 can be well fitted by a constant synchrotron spectrum, while in the case of II Zw 40 it is only at the lowest frequencies that nonthermal emission becomes evident. We therefore decided to fit a power-law only to its high-frequency data, obtaining an optically thin thermal spectrum with slope $\alpha_{\text {th }}=0.10 \pm 0.02$. This spectrum is then extrapolated to the lower frequencies at which we retrieve the nonthermal flux densities by subtracting the extrapolation from the measured fluxes. In Fig. 1 the dashed cyan line shows the fit to the three high-frequency data, yielding a perfect optically thin thermal spectrum. At the two low frequencies we have subtracted the extrapolated thermal flux densities from those observed, such as to yield the nonthermal spectrum.

We have also produced fits to the spectrum of IIZw70 in which $\alpha_{\text {nth }}$ is limited to $1.0,0.9,0.8,0.7$, and 0.6 . We note that $\alpha_{\text {nth }}$ may take values as low as 0.8 , and the total fit is still compatible with the error bars of the observational data. The thermal spectrum is a little lower in this case and, as expected, $\chi_{v}^{2}$ is worse. Values of 0.7 and 0.6 cause the total fit to go below the first data point. We also tried to fit with maximum values of 0.5 and 0.4 , but the fits did not converge.

Obviously, the BCDGs and IC 10 are dominated by the thermal radio emission $\left(f_{\text {th }} \geq 0.2\right.$ at $\left.1 \mathrm{GHz}\right)$ meaning that we cannot draw any firm conclusions about the shape of the synchrotron spectra at all.

Also for NGC 3079 a straight or curved synchrotron spectrum formally gives the best fit, and for NGC 4038, all four fits are acceptable. However, the thermal flux density obtained for the constant/curved fits are rather low. Compared to the $\mathrm{H} \alpha$ data they would require internal extinctions of 2 . 5 (for NGC 3079) and 1 . 0 (for NGC 4038) which seems rather low for these edgeon, highly extinguished galaxies.

For 10 of the 14 galaxies, the best-fit spectrum is that with either a break or a cutoff. For 5 galaxies, the $\chi_{v}^{2}$ values are very similar in both cases (within a factor of 1.5), and for all 10 galaxies both break and cutoff give acceptable fits. In addition, the broad radio range covered by our data in most of these galaxies renders both the steepening of the spectrum starting at $\sim 1-5 \mathrm{GHz}$ due to the strong curvature of the synchrotron emission, and the subsequent flattening due to the dominance of the thermal radio emission (above $\sim 10 \mathrm{GHz}$ ) visible in the measured spectra. The most noticeable cases are NGC 2146, NGC 4449, NGC 4631, and NGC 5194; but also in NGC 1569, NGC 3034, and NGC 3310 this trend is visible. The fact that we directly observe the steepening and subsequent flattening in the data lends support for the reality of the pronounced curvature of the synchrotron spectra that our fitting procedure yields.

\section{Discussion}

\subsection{Comparison of thermal radio and $\mathrm{H} \alpha$ emission}

We have used the thermal flux densities resulting from our spectral decomposition to compare them with what is predicted from the observed $\mathrm{H} \alpha$ fluxes corrected for $\mathrm{N}$ II emission and Galactic extinction (Table 1). We used the relation derived by Lequeux (1980), converted from $\mathrm{H} \beta$ to $\mathrm{H} \alpha$ :

$S_{\mathrm{th}, \mathrm{H} \alpha}=1.14 \times 10^{12}\left(\frac{v}{\mathrm{GHz}}\right)^{-0.1}\left(\frac{T_{\mathrm{e}}}{10^{4} \mathrm{~K}}\right)^{0.34}\left[\frac{F(\mathrm{H} \alpha)}{\mathrm{erg} \mathrm{s}^{-1} \mathrm{~cm}^{-2}}\right] \mathrm{mJy}$.

The extinction is then calculated via the equation

$A(\mathrm{H} \alpha)=-2.5 \log \left(\frac{S_{\mathrm{th}, \mathrm{H} \alpha}}{S_{\mathrm{th}, \mathrm{fit}}}\right)$.

Since extinction is caused by the dust in the galaxy planes, we have plotted in Fig. 2 the extinction resulting from Eq. (18) versus the metallicity, for which we have collected the quantity $12+\log (\mathrm{O} / \mathrm{H})$ from the literature (Table 1$)$. As is to be expected, there is a trend of increasing extinction with increasing metallicity, with the highly inclined spiral galaxies having the largest extinctions. Dwarf galaxies are known to possess lower metallicities and thus have a low dust content, hence they are found in the lower left part of the diagram. The values for IC 10 and II Zw 40 are even negative, which is most likely due to an overestimate of the Galactic extinction which is very high in both 
U. Klein et al.: Synchrotron spectra of galaxies

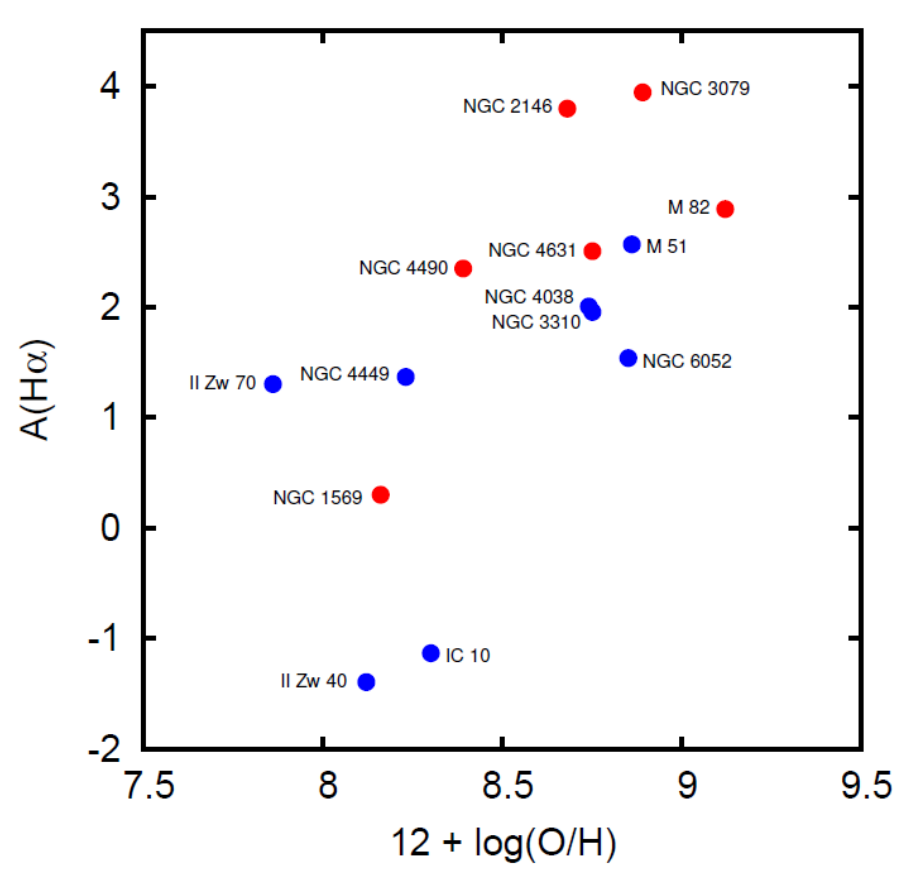

Fig. 2. $\mathrm{H} \alpha$ extinction vs. metallicity. Highly inclined ( $>75$ degrees) and edge-on galaxies are marked with red dots.

cases (3. 4 for IC 10 and 1.8 for II Zw 40). An underestimate of the thermal radio emission is unlikely in these two cases because for II Zw 40 the thermal radio emission is directly given by the high-frequency data and for IC 10 the result for the thermal fraction yields very similar values for all fits.

\subsection{Thermal fraction}

In Fig. 3 we show the fraction of thermal emission at $1 \mathrm{GHz}$ resulting from our spectral fits, plotted against the $K$-band luminosity of the galaxies. Since this luminosity is a measure for the stellar mass, the diagram clearly indicates a decreasing relative amount of nonthermal radiation as we move to small stellar masses. In fact, the left half of this plot contains all the dwarf galaxies in our sample. This result corroborates the early conjecture of Klein et al. (1991) that the lowest-mass galaxies are unable to retain the recently produced cosmic rays. Alternatively, the high thermal fraction in the most extreme galaxies, II Zw 40 and II $\mathrm{Zw} 70$, might be the result of a temporal effect. Both galaxies are currently experiencing a strong and very recent starburst, with an age of only 3-5 Myr, derived from the presence of Wolf-Rayet feature in their spectra. This means that the newly formed ionising stars are contributing to the thermal radio emission, but no supernovae may have gone off yet. Hence, the observed synchrotron radiation may have been produced during a previous epoch of star formation. We also compare the thermal fraction with the SFR (from Table 1) but find no correlation.

It is important to note here that for some of the galaxies in our sample the amount of thermal emission resulting from our analysis is higher than thought hitherto, which has consequences for any conjectures based upon the thermal radio continuum in star forming galaxies. This is particularly true for higher frequencies where the cutoff/break lowers the synchrotron emission considerably. At $10 \mathrm{GHz}$, the mean thermal fraction for the galaxies is 0.6 - considerably more than what has been believed so far.

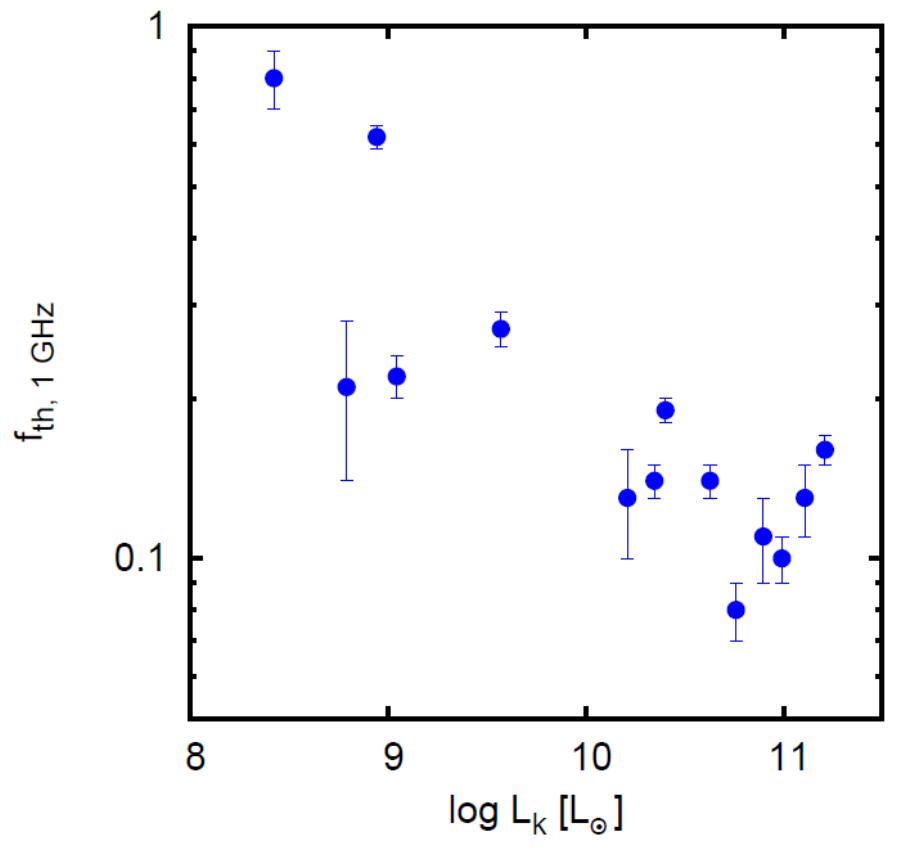

Fig. 3. Fraction of thermal emission at $1 \mathrm{GHz}$ vs. $K$-band luminosity, the errors resulting from our fits (see Table 3 ).

\subsection{Injection spectra}

In Fig. 4 a superposition of the low-frequency spectral indices of the synchrotron radiation of our galaxy sample with the spectral indices of Galactic supernova remnants (SNR) is shown, the latter data taken from the catalogue of Green (2014). The two distributions are rather similar, albeit with statistics that are vastly different (203 vs. 14 objects). The resulting values of the mean and standard deviation are $\left\langle\alpha_{\mathrm{SNR}}\right\rangle=0.50, \sigma_{\alpha_{\mathrm{SNR}}}=0.33$ and $\left\langle\alpha_{\text {Gal }}\right\rangle=0.59, \sigma_{\alpha_{\text {Gal }}}=0.20$ for the SNR and galaxies, respectively. Both the mean and the rms are rather similar, possibly suggesting that the synchrotron spectra that we measure at low radio frequencies reflect the injection spectra of the SNR.

An outlier is II $\mathrm{Zw} 70$, which has a low-frequency spectral index of $\alpha_{\text {nth }}=1.15 \pm 0.12$. Such a steep spectrum is indicative of the impact of synchrotron and inverse-Compton losses which have steepened the injection spectral index by +0.5 . It is unclear why II $\mathrm{Zw} 70$ is so different from the rest of the galaxies. The SFR per $L_{\mathrm{K}}$, which can be taken as a measure of the capability of the galaxy to expell material into the halo, is similar to that of the other starburst galaxies, such as M 82 or NGC 1569 (but more than a factor of 10 lower than for the otherwise similar BCDG II Zw 40).

\subsection{Spectral breaks and cutoffs}

For the majority of our galaxies, the fitting of their radio spectra shows the need for a break in the synchrotron spectra in the range of 1-12 GHz, corresponding to particle energies of 1.5$7 \mathrm{GeV}$, depending on the magnetic-field strengths (see Table 4). The low-frequency spectrum in all cases except II Zw 70 has a spectral index in the range of what is found for SNRs, suggesting that the relativistic electrons emitting in this range have not suffered any significant synchrotron and inverse-Compton losses that would steepen their spectrum. The steep high-frequency spectrum indicates that synchrotron or inverse-Compton losses are important (in the case of a break) or a complete lack of relativistic electrons above $\sim 5 \mathrm{GeV}$ (in the case of a cutoff). 


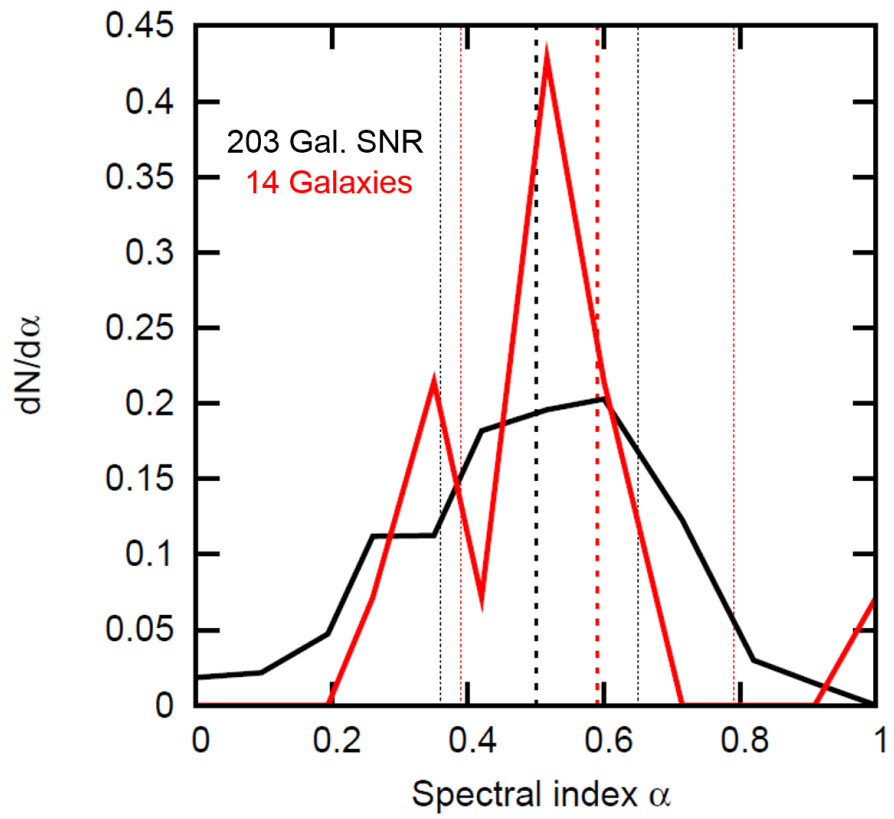

Fig. 4. Histogram of the spectral indices of the low-frequency synchrotron radiation of our sample galaxies (red) and of Galactic supernova remnants (black). The vertical dashed lines indicate the mean (thick) and variance (thin) of each distribution.

Table 4. Radio sizes, magnetic fields, and cutoff/break energies.

\begin{tabular}{|c|c|c|c|c|}
\hline Galaxy & $\begin{array}{c}\text { Radio size } \\
{[\mathrm{kpc}]}\end{array}$ & $\begin{array}{c}B^{1} \\
{[\mu \mathrm{G}]}\end{array}$ & $\begin{array}{c}E \\
{[\mathrm{GeV}]}\end{array}$ & Shape \\
\hline II Zw 40 & 0.8 & 29 & - & - \\
\hline & $<0.6$ & $<40$ & - & constant \\
\hline IC 10 & 1.3 & 14 & - & constant \\
\hline NGC 1569 & 2.5 & 16 & 6.8 & cutoff \\
\hline NGC 4449 & 5.0 & 14 & 4.6 & cutoff \\
\hline$\overline{\mathrm{NGC}} \overline{4} \overline{490}$ & 10 & 20 & 4.6 & cutoff \\
\hline $\mathrm{N}$ & & 13 & & cutoff \\
\hline NGC 5194 & 15 & 18 & 5.0 & cutoff \\
\hline NGC 4038 & 18 & 27 & - & break \\
\hline NGC 6052 & 4.0 & 71 & - & break \\
\hline$\overline{\mathrm{NGC}} 2 \overline{14} \overline{6}$ & $10^{-}$ & 40 & 3.1 & cutoff \\
\hline NGC 3034 & 1.7 & 66 & 3.3 & cutoff \\
\hline NGC 3079 & 13 & 32 & - & break \\
\hline NGC 3310 & 4.4 & 39 & - & break \\
\hline
\end{tabular}

Notes. ${ }^{(1)}$ The magnetic field is calculated with the minimum energy assumption.

As outlined in Sect. 4.1, a sharp break can be explained in an open box model with convection. The break is produced because high-energy electrons, emitting at frequencies above the break, suffer substantial synchrotron or inverse-Compton losses before they arrive at the edge of the halos, whereas low-energy electrons do not. We can estimate the half-lifetime of the relativistic electrons emitting the synchrotron radiation at the break frequency, which is determined by synchrotron and inverse-Compton losses (see, e.g. Klein \& Fletcher 2015):

$t_{1 / 2}=1.25 \times 10^{10} \cdot\left[\left(\frac{B}{\mu \mathrm{G}}\right)^{2}+\left(\frac{B_{\mathrm{eq}}}{\mu \mathrm{G}}\right)^{2}\right]^{-1} \cdot\left(\frac{E}{\mathrm{GeV}}\right)^{-1} \mathrm{yr}$.
Here, $E$ is the energy of the relativistic particles, $B$ is the total magnetic-field strength, and $B_{\text {eq }}$ is the field strength equivalent to the energy density of the local radiation field. The latter can be obtained via

$B_{\mathrm{eq}}=\sqrt{\frac{32}{c}} \cdot \frac{L_{\mathrm{bol}}^{1 / 2}}{d}$.

Here, $L_{\text {bol }}$ is the bolometric luminosity of a region of size $d$. Kennicutt et al. (2007) have measured the quantity $v \cdot L_{v}$ at $24 \mu \mathrm{m}$ (which approximates the luminosity) for a number of $\mathrm{H}$ II regions in M 51, with typical values of $10^{41} \mathrm{erg} \mathrm{s}^{-1}$, obtained with aperture sizes of $13^{\prime \prime}$ (hence, $d=500 \mathrm{pc}$ ). Plugging this into Eq. (20), we obtain $B_{\text {eq }} \approx 7 \mu \mathrm{G}$. The strength of the magnetic field in our sample galaxies is between $B=10 \mu \mathrm{G}$ and $B=70 \mu \mathrm{G}$ (Table 4). This implies a range for the particle halflifetimes of between $7.5 \times 10^{5} \mathrm{yr}$ and $2.5 \times 10^{7} \mathrm{yr}(\mathrm{Tev}$ and $\mathrm{PeV}$ particles enter the $\mathrm{GeV}$ regime on time scales much shorter than any dynamical time scales under conditions considered above). Assuming a vertical halo size of about $250 \mathrm{pc}$, this implies convection speeds of between 10 and $300 \mathrm{~km} \mathrm{~s}^{-1}$, which is reasonable. In order to test this interpretation, it would be useful to carry out a similar analysis for quiescent galaxies with a low SFR per area for which we would expect much lower vertical propagation of the relativistic electrons.

A cutoff in the relativistic electron distribution is more difficult to explain. Energy losses can produce a cutoff only in a single-injection scenario where radiative ageing depopulates the high-energy part of the relativistic electron distribution with time. This situation is unrealistic for the integrated radio emission of an entire galaxy. The acceleration process of cosmic rays in SNRs is effective up to $\mathrm{TeV}$ energies and the synchrotron spectra of young SNRs can be observed up to X-ray energies. At high energies, the short synchrotron energy loss time in the strong magnetic fields $(>100 \mu \mathrm{G})$ in SNR (Völk et al. 2005) is expected to produce a steepening of the electron injection spectrum. This can be seen from Eq. (19), which for a particle energy of, for example, $100 \mathrm{GeV}$ in a $100 \mu \mathrm{G}$ magnetic field yields a lifetime of $\sim 10^{4} \mathrm{yr}$. This is comparable to the Sedov phase of a SNR $\left(\tau \sim 3 \times 10^{4} \mathrm{yr}\right)$ meaning that at energies above $\sim 100 \mathrm{GeV}$ synchrotron cooling should be relevant and produce a steeper injection spectrum above $\sim 2 \times 10^{13} \mathrm{~Hz}$. However, the breaks/cutoffs that we infer are at much lower particle energies and do therefore not probe this process. Thus, in the framework of the simple models considered here, it is unclear which process could produce a cutoff in the synchrotron spectrum. It is, however, obvious that this must have to do with the relative time scales of energy gain, loss and escape of the relativistic electrons, as discussed by Schlickeiser (1984).

\section{Summary and conclusions}

We have analysed the radio continuum spectra of 14 star-forming galaxies by fitting nonthermal (synchrotron) and thermal (freefree) radiation laws to carefully selected measurements, covering a frequency range of $\sim 300 \mathrm{MHz}$ to $24.5 \mathrm{GHz}(32 \mathrm{GHz}$ in case of M 82). The 24.5-GHz measurements, mostly unpublished to date, are crucial for a more reliable separation of the thermal and nonthermal components in this analysis.

We find that the majority of the synchrotron spectra are not simple power-laws as believed hitherto. The curved shape of the synchrotron spectrum is clearly visible in many of the total radio spectra, which steepen over a range of several $\mathrm{GHz}$ and flatten at higher frequencies due to the thermal radio emission. Our fitting shows that the lowest values of the reduced $\chi_{v}^{2}$ result for 
synchrotron spectra in a mean slope $\alpha_{\text {nth }}=0.59 \pm 0.20$ in the low-frequency regime, and a break or an exponential decline in the frequency range of $1-12 \mathrm{GHz}$. There is only one galaxy that shows pure power-laws (the dwarf galaxy IC 10). In the case of the BCDGs, II Zw 40 and II Zw 70, only the slope (and not the shape) of the synchrotron spectrum can be deciphered, since a deviation from a purely thermal spectrum is only evident at the lowest frequencies involved.

For the bulk of the sample galaxies, simple power-laws or mildly curved synchrotron spectra lead to unrealistic low thermal flux densities (frequently $S_{\text {th }}=0$ ), and/or to strong deviations from the expected optically thin free-free spectra with slope $\alpha_{\text {th }}=0.10$ in the fits. Assuming energy equipartition between relativistic particles and magnetic fields, the cutoff and break frequencies translate into energies in the range of $1.5-7 \mathrm{GeV}$. The average spectral index of the low-frequency spectra obtained here is comparable to that found for Galactic (shell-type) supernova remnants.

A comparison of the thermal flux densities resulting from our fits with the (foreground-corrected) $\mathrm{H} \alpha$ fluxes yields the extinction, which increases with metallicity. The fraction of thermal emission at $1 \mathrm{GHz}$ is higher in some of our galaxies than believed hitherto, and the discrepancy increases towards higher frequencies where the mean thermal fraction is $\sim 0.6$. It is highest in the dwarf galaxies of our sample, which we interpret either in terms of a lack of containment of synchrotron emission in these low-mass systems, or alternatively, in the case of II Zw 40 and II Zw 70, as a time effect due to a very young starburst.

The asymptotic low-frequency synchrotron spectra derived here provide a firm "leverage" for low-frequency studies, for example, with LOFAR. In order to significantly increase the number of radio continuum spectra of galaxies allowing analyses as presented here, expensive mapping with large single-dish telescopes (Effelsberg, GBT, Sardinia Radio Telescope) in the frequency range of 25-40 GHz (so-called Ka-band in radio astronomy) is indispensible.

An interpretation of the rapid declines in the synchrotron spectra (break or exponential cutoff) at $\mathrm{GeV}$ particle energies is not obvious, since the relativistic particles gain energies up to the $\mathrm{TeV}$ range within the supernovae. In a simple model, a convective wind carrying away the low-energy relativistic electrons could explain a sharp break. The break energy must depend on the relative time scales of energy gain (acceleration), loss (synchrotron and inverse-Compton) and escape of the relativistic electrons.

Acknowledgements. We wish to thank H. Lesch and R. Schlickeiser for helpful discussions. U.K. acknowledges financial support by the German Deutsche Forschungsgemeinschaft, DFG project FOR 1254, and is very grateful for the kind hospitality at the Departamento de Física Teórica y del Cosmos, Universidad de Granada. U.L. and S.V. acknowledge support by the research projects AYA2014-53506-P from the Spanish Ministerio de Economía y Competitividad, from the European Regional Development Funds (FEDER) and the Junt de Andalucía (Spain) grants FQM108. This research has made use of the NASA/IPAC Extragalactic Database (NED), which is operated by the Jet Propulsion Laboratory, California Institute of Technology, under contract with the $\mathrm{Na}$ tional Aeronautics and Space Administration. We also acknowledge the use of the HyperLeda database (http://leda.univ-lyon $1 . \mathrm{fr}$ ). This research made use of ASTROPY, a community-developed core PYTHON (http:// WWw . python.org) package for Astronomy (Astropy Collaboration et al. 2013) IPYTHON (Pérez \& Granger 2007); MATPLOTLIB (Hunter 2007); NUMPY (van der Walt et al. 2011); SCIPY (Jones et al. 2001). Finally, we are very grateful to the referee for her/his comments, which helped to improve the manuscript.

\section{References}

Adebahr, B., Krause, M., Klein, U., et al. 2013, A\&A, 555, A23
Astropy Collaboration, Robitaille, T. P., Tollerud, E. J., et al. 2013, A\&A, 558, A33

Baars, J. W. M., Genzel, R., Pauliny-Toth, I. I. K., \& Witzel, A. 1977, A\&A, 61, 99

Balkowski, C., Chamaraux, P., \& Weliachew, L. 1978, A\&A, 69, 263

Bastian, N., Trancho, G., Konstantopoulos, I. S., \& Miller, B. W. 2009, ApJ, 701, 607

Basu, A., Mao, S. A., Kepley, A. A., et al. 2017, MNRAS, 464, 1003

Beck, R., Klein, U., \& Wielebinski, R. 1987, A\&A, 186, 95

Becker, R. H., White, R. L., \& Edwards, A. L. 1991, ApJS, 75, 1

Berezhko, E. G., \& Völk, H. J. 1997, Astropart. Phys., 7, 183

Branch, M. A., Coleman, T. F., \& Li, Y. 1999, SIAM J. Sci. Comput., 21, 1

Braun, R., Oosterloo, T. A., Morganti, R., Klein, U., \& Beck, R. 2007, A\&A, 461,455

Brown, M. J. I., Moustakas, J., Smith, J.-D. T., et al. 2014, ApJS, 212, 18

Chyży, K. T., \& Beck, R. 2004, A\&A, 417, 541

Chyży, K. T., Beck, R., Kohle, S., Klein, U., \& Urbanik, M. 2000, A\&A, 355, 128

Chyży, K. T., Knapik, J., Bomans, D. J., et al. 2003, A\&A, 405, 513

Chyży, K. T., Weżgowiec, M., Beck, R., \& Bomans, D. J. 2011, A\&A, 529, A94

Chyży, K. T., Drzazga, R. T., Beck, R., et al. 2016, ApJ, 819, 39

Clemens, M. S., Alexander, P., \& Green, D. A. 1999, MNRAS, 307, 481

Condon, J. J. 1987, ApJS, 65, 485

Condon, J. J., Cotton, W. D., Greisen, E. W., et al. 1998, AJ, 115, 1693

Dale, D. A., Cohen, S. A., Johnson, L. C., et al. 2009, ApJ, 703, 517

de Bruyn, A. G. 1977, A\&A, 58, 221

de Jong, M. L. 1967, ApJ, 150, 1

Deeg, H.-J., Brinks, E., Duric, N., Klein, U., \& Skillman, E. 1993, ApJ, 410, 626

Drury, L. O., Aharonian, F. A., \& Voelk, H. J. 1994, A\&A, 287, 959

Dumke, M., Krause, M., Wielebinski, R., \& Klein, U. 1995, A\&A, 302, 691

Duric, N., \& Seaquist, E. R. 1988, ApJ, 326, 574

Duric, N., Seaquist, E. R., Crane, P. C., Bignell, R. C., \& Davis, L. E. 1983, ApJ, 273, L11

Duric, N., Seaquist, E. R., Crane, P. C., \& Davis, L. E. 1986, ApJ, 304, 82

Duric, N., Bourneuf, E., \& Gregory, P. C. 1988, AJ, 96, 81

Ekers, R. D., \& Sancisi, R. 1977, A\&A, 54, 973

Emerson, D. T., Klein, U., \& Haslam, C. G. T. 1979, A\&A, 76, 92

Engelbracht, C. W., Rieke, G. H., Gordon, K. D., et al. 2008, ApJ, 678, 804

Fletcher, A., Beck, R., Shukurov, A., Berkhuijsen, E. M., \& Horellou, C. 2011, MNRAS, 412, 2396

Gil de Paz, A., Madore, B. F., \& Pevunova, O. 2003, ApJS, 147, 29

Gioia, I. M., \& Gregorini, L. 1980, A\&AS, 41, 329

Gioia, I. M., Gregorini, L., \& Klein, U. 1982, A\&A, 116, 164

Golla, G. 1999, A\&A, 345, 778

Green, D. A. 2014, Bull. Astron. Soc. India, 42, 47

Gregory, P. C., \& Condon, J. J. 1991, ApJS, 75, 1011

Griffith, M. R., Wright, A. E., Burke, B. F., \& Ekers, R. D. 1994, ApJS, 90,179

Haynes, R. F., Huchtmeier, W. K. G., \& Siegman, B. C. 1975, A compendium of radio measurements of bright galaxies (Melbourne: CSIRO)

Heidmann, J. 1979, in Extragalactic Astronomy - Meeting "Sol-Espace", ed. C. Balkowski-Mauger, 205

Heidmann, J., Klein, U., \& Wielebinski, R. 1982, A\&A, 105, 188

Holtzman, J. A., Watson, A. M., Mould, J. R., et al. 1996, AJ, 112, 416

Hummel, E. 1980, A\&AS, 41, 151

Hummel, E. 1991, A\&A, 251, 442

Hummel, E., \& Dettmar, R.-J. 1990, A\&A, 236, 33

Hummel, E., Pedlar, A., Davies, R. D., \& van der Hulst, J. M. 1985, A\&AS, 60, 293

Hummel, E., Beck, R., \& Dahlem, M. 1991, A\&A, 248, 23

Hunter, J. D. 2007, Comput. Sci. Eng., 9, 90

Irwin, J. A., \& Saikia, D. J. 2003, MNRAS, 346, 977

Irwin, J., Beck, R., Benjamin, R. A., et al. 2012, AJ, 144, 44

Israel, F. P., \& de Bruyn, A. G. 1988, A\&A, 198, 109

Israel, F. P., \& Mahoney, M. J. 1990, ApJ, 352, 30

Israel, F. P., \& van der Hulst, J. M. 1983, AJ, 88, 1736

Jaffe, W. J., \& Perola, G. C. 1973, A\&A, 26, 423

Jaffe, W. J., Perola, G. C., \& Tarenghi, M. 1978, ApJ, 224, 808

Jarrett, T. H., Chester, T., Cutri, R., et al. 2000, AJ, 119, 2498

Jarrett, T. H., Chester, T., Cutri, R., Schneider, S. E., \& Huchra, J. P. 2003, AJ, 125,525

Jones, E., Oliphant, T., Peterson, P., et al. 2001, SciPy: Open source scientific tools for Python, http: //www. scipy.org/

Kardashev, N. S. 1962, SvA, 6, 317

Kazès, I., Le Squeren, A. M., \& Nguyen-Quang-Rieu. 1970, Astrophys. Lett., 6, 193

Kehrig, C., Vílchez, J. M., Sánchez, S. F., et al. 2008, A\&A, 477, 813

Kellermann, K. I., Pauliny-Toth, I. I. K., \& Williams, P. J. S. 1969, ApJ, 157, 1

Kennicutt, Jr., R. C., Calzetti, D., Walter, F., et al. 2007, ApJ, 671, 333 
Kennicutt, Jr., R. C., Hao, C.-N., Calzetti, D., et al. 2009, ApJ, 703, 1672

Kennicutt, Jr., R. C., Lee, J. C., Funes, José G., S. J., Sakai, S., \& Akiyama, S. 2008, ApJS, 178, 247

Kepley, A. A., Mühle, S., Everett, J., et al. 2010, ApJ, 712, 536

Klein, U. 1983, A\&A, 121, 150

Klein, U. 1988, Ph.D. Thesis, Habilitation Thesis, Univ. Bonn

Klein, U., \& Emerson, D. T. 1981, A\&A, 94, 29

Klein, U., \& Fletcher, A. 2015, Galactic and Intergalactic Magnetic Fields (Springer)

Klein, U., \& Gräve, R. 1986, A\&A, 161, 155

Klein, U., Gräve, R., \& Wielebinski, R. 1983, A\&A, 117, 332

Klein, U., Wielebinski, R., \& Beck, R. 1984a, A\&A, 135, 213

Klein, U., Wielebinski, R., \& Thuan, T. X. 1984b, A\&A, 141, 241

Klein, U., Wielebinski, R., \& Morsi, H. W. 1988, A\&A, 190, 41

Klein, U., Weiland, H., \& Brinks, E. 1991, A\&A, 246, 323

Klein, U., Hummel, E., Bomans, D. J., \& Hopp, U. 1996, A\&A, 313, 396

Kobulnicky, H. A., \& Skillman, E. D. 1997, ApJ, 489, 636

Kregel, M., \& Sancisi, R. 2001, A\&A, 376, 59

Lequeux, J. 1971, A\&A, 15, 30

Lequeux, J. 1980, in Saas-Fee Advanced Course 10: Star Formation, eds A. Maeder, \& L. Martinet, 9999

Lisenfeld, U., Alexander, P., Pooley, G. G., \& Wilding, T. 1996, MNRAS, 281, 301

Lisenfeld, U., Wilding, T. W., Pooley, G. G., \& Alexander, P. 2004, MNRAS, 349,1335

Maehara, H., Inoue, M., Takase, B., \& Noguchi, T. 1985, PASJ, 37, 451

Magrini, L., \& Gonçalves, D. R. 2009, MNRAS, 398, 280

Mao, S. A., Zweibel, E., Fletcher, A., Ott, J., \& Tabatabaei, F. 2015, ApJ, 800, 92

Marvil, J., Owen, F., \& Eilek, J. 2015, AJ, 149, 32

McCutcheon, W. H. 1973, AJ, 78, 18

Mora, S. C., \& Krause, M. 2013, A\&A, 560, A42

Moustakas, J., \& Kennicutt, Jr., R. C. 2006, ApJS, 164, 81

Moustakas, J., Kennicutt, Jr., R. C., Tremonti, C. A., et al. 2010, ApJS, 190, 233

Mulcahy, D. D., Horneffer, A., Beck, R., et al. 2014, A\&A, 568, A74
Neininger, N. 1992, A\&A, 263, 30

Nikiel-Wroczyński, B., Jamrozy, M., Soida, M., Urbanik, M., \& Knapik, J. 2016, MNRAS, 459, 683

Niklas, S., Klein, U., Braine, J., \& Wielebinski, R. 1995, A\&AS, 114, 21

Niklas, S., Klein, U., \& Wielebinski, R. 1997, A\&A, 322, 19

Pérez, F., \& Granger, B. E. 2007, Comput. Sci. Eng., 9, 21

Perley, R. A., \& Butler, B. J. 2013, ApJS, 204, 19

Pfleiderer, J., Durst, C., \& Gebler, K.-H. 1980, MNRAS, 192, 635

Pilyugin, L. S., \& Thuan, T. X. 2007, ApJ, 669, 299

Purkayastha, A. 2014, Ph.D. Thesis, University Bonn

Rengelink, R. B., Tang, Y., de Bruyn, A. G., et al. 1997, A\&AS, 124, 259

Robertson, P., Shields, G. A., Davé, R., Blanc, G. A., \& Wright, A. 2013, ApJ, 773, 4

Sage, L. J., Loose, H. H., \& Salzer, J. J. 1993, A\&A, 273, 6

Schlickeiser, R. 1984, A\&A, 136, 227

Segalovitz, A. 1977, A\&A, 54, 703

Skillman, E. D., \& Klein, U. 1988, A\&A, 199, 61

Slee, O. B. 1995, Austr. J. Phys., 48, 143

Sramek, R. 1975, AJ, 80, 771

Srivastava, S., Kantharia, N. G., Basu, A., Srivastava, D. C., \& Ananthakrishnan, S. 2014, MNRAS, 443, 860

Sulentic, J. W. 1976, ApJS, 32, 171

Tabatabaei, F. S., Schinnerer, E., Krause, M., et al. 2017, ApJ, 836, 185

Thuan, T. X., \& Izotov, Y. I. 2005, ApJS, 161, 240

Tovmassian, H. M. 1968, Austr. J. Phys., 21, 193

van der Hulst, J. M. 1979, A\&A, 71, 131

van der Kruit, P. C., \& de Bruyn, A. G. 1976, A\&A, 48, 373

van der Walt, S., Colbert, S. C., \& Varoquaux, G. 2011, Comput. Sci. Eng., 13, 22

Varenius, E., Conway, J. E., Martí-Vidal, I., et al. 2015, A\&A, 574, A114

Viallefond, F., Allen, R. J., \& de Boer, J. A. 1980, A\&A, 82, 207

Völk, H. J., Berezhko, E. G., \& Ksenofontov, L. T. 2005, A\&A, 433, 229

Werner, W. 1988, A\&A, 201, 1

Wielebinski, R., \& von Kap-Herr, A. 1977, A\&A, 59, L17

Williams, P. K. G., \& Bower, G. C. 2010, ApJ, 710, 1462

Wynn-Williams, C. G., \& Becklin, E. E. 1986, ApJ, 308, 620 
Appendix A: Final spectra
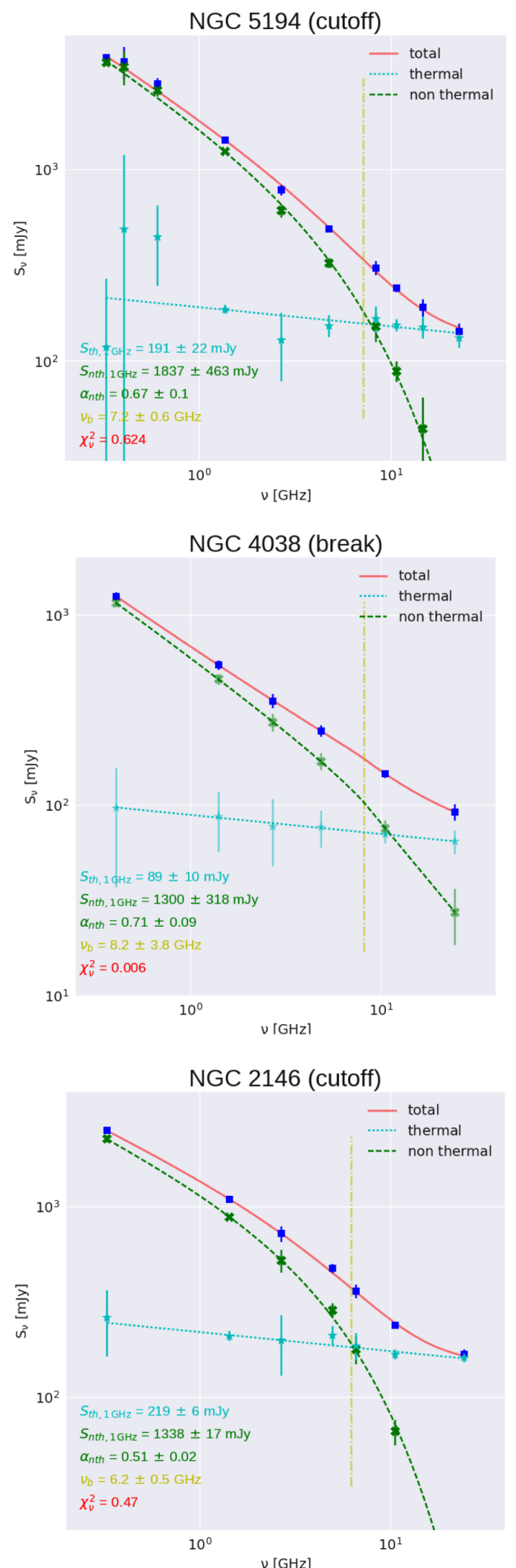
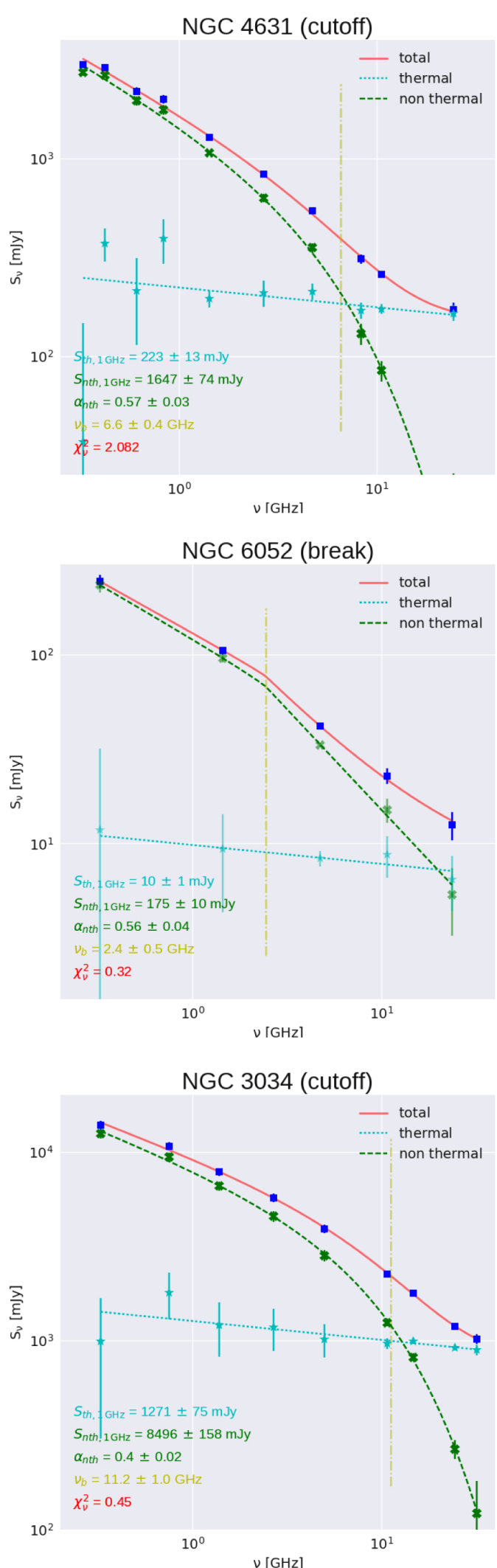

Fig. A.1. Radio continuum spectra of the sample galaxies. Except for II Zw 40 (see text for the procedure and plotted lines here), the plots show the following: measured flux densities (blue squares), best fit of the radio continuum spectrum (solid red line). The best parameters are listed in the lower left part of the figure along with the reduced chi-square $\left(\chi_{v}^{2}\right)$. The free-free (thermal) and synchrotron (nonthermal) components of the models are depicted by the dotted cyan line and dashed green line, respectively. The green crosses represent the observed nonthermal component (i.e. the modelled thermal component removed from the observational data). The cyan stars delineate the observed thermal component (i.e. the modelled nonthermal component subtracted from the observational data). The vertical yellow dash-dotted line marks the break frequency $v_{\mathrm{b}}$. (Continuation of Fig. 1). 

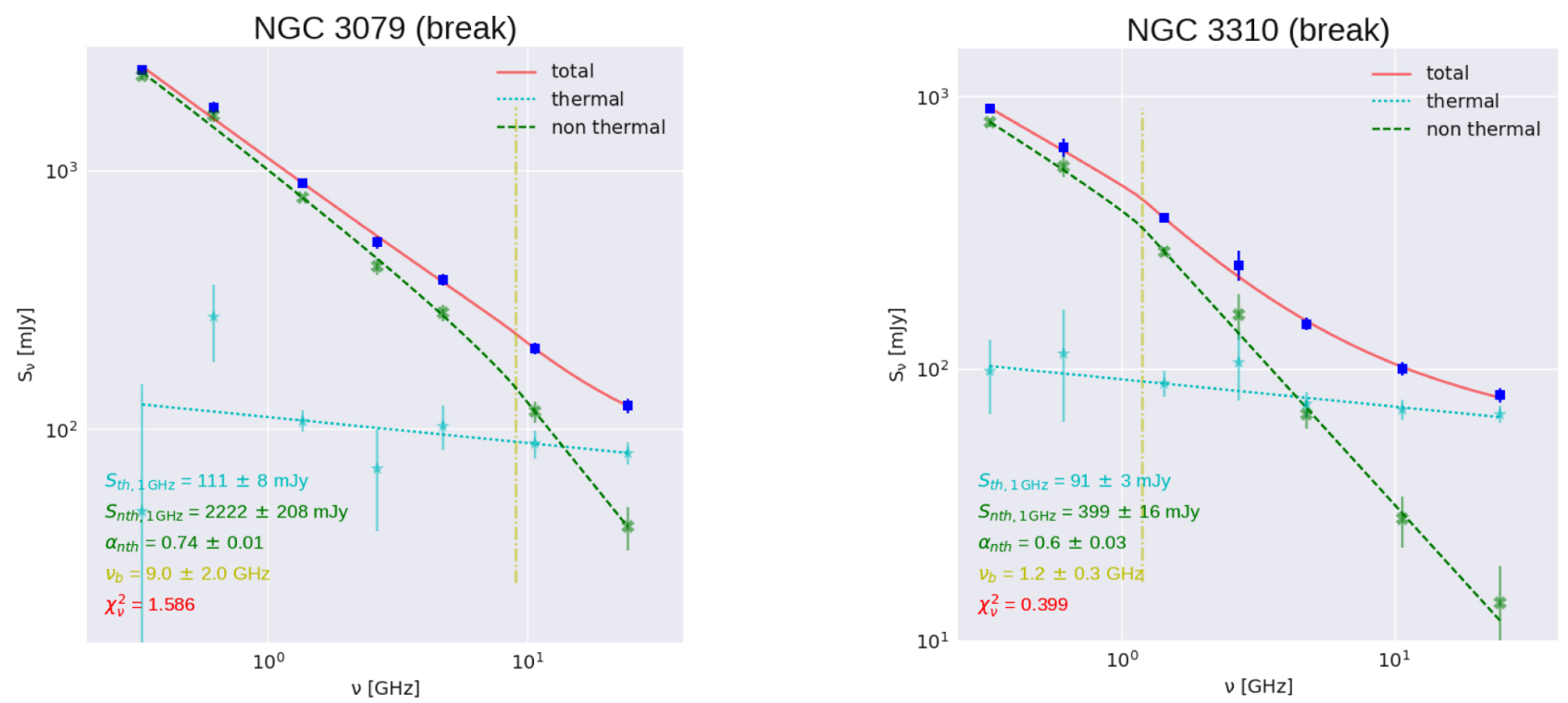

Fig. A.1. continued.

\section{Appendix B: Flux densities}

Table B.1. II Zw 40.

\begin{tabular}{rrrl}
\hline \hline Frequency & Flux density & Error & Reference \\
\hline 0.325 & 38 & 4 & Deeg et al. (1993) \\
1.459 & 30.1 & 0.4 & Klein et al. (1991), Deeg et al. (1993) \\
4.88 & 21.5 & 1.9 & Jaffe et al. (1978), Klein et al. (1984b), Klein et al. (1991) \\
10.63 & 20.1 & 1.5 & Skillman \& Klein (1988), Klein et al. (1984b) \\
24.500 & 18 & 4 & Klein et al. (1984b) \\
\hline
\end{tabular}

Table B.2. II Zw 70.

\begin{tabular}{rrrl}
\hline \hline Frequency & Flux density & Error & Reference \\
0.327 & 11.2 & 2.0 & Skillman \& Klein (1988) \\
0.609 & 6.5 & 0.8 & Skillman \& Klein (1988) \\
1.440 & 4.56 & 0.34 & Wynn-Williams \& Becklin (1986), Balkowski et al. (1978) \\
4.795 & 3.04 & 0.13 & Klein et al. (1984b), Wynn-Williams \& Becklin (1986), Skillman \& Klein (1988) \\
10.700 & 2.73 & 0.20 & Skillman \& Klein (1988), Klein et al. (1984b)
\end{tabular}

Table B.3. IC 10

\begin{tabular}{rrrl}
\hline \hline Frequency & Flux density & Error & Reference \\
\hline 0.327 & 352 & 20 & WENSS, this work \\
1.43 & 377 & 6 & this work \\
2.64 & 283 & 20 & Chyży et al. (2011) (recomputed) \\
4.82 & 219 & 8 & Klein et al. (1983), Klein \& Gräve (1986), Becker et al. (1991) \\
10.575 & 162 & 8 & Klein \& Gräve (1986), Chyży et al. (2003) \\
24.5 & 118 & 18 & Klein \& Gräve (1986) \\
\hline
\end{tabular}

Table B.4. NGC 1569.

\begin{tabular}{rrrl}
\hline \hline Frequency & Flux density & Error & Reference \\
\hline 0.350 & 750 & 20 & Purkayastha (2014), Ph.D. thesis, Univ. Bonn \\
0.610 & 610 & 20 & Israel \& de Bruyn (1988) \\
1.415 & 425 & 20 & Hummel (1980), Israel \& de Bruyn (1988) \\
2.700 & 318 & 20 & Pfleiderer et al. (1980), Sulentic (1976) \\
4.800 & 233 & 20 & Klein \& Gräve (1986), Gregory \& Condon (1991) \\
8.350 & 176 & 5 & this work \\
10.700 & 155 & 10 & Klein \& Gräve (1986) \\
15.36 & 116 & 13 & Lisenfeld et al. (2004) \\
24.500 & 96 & 8 & Klein \& Gräve (1986) \\
\hline
\end{tabular}


Table B.5. NGC 2146 .

\begin{tabular}{rrrl}
\hline \hline Frequency & Flux density & Error & Reference \\
\hline 0.327 & 2520 & 100 & WENSS, this work \\
1.43 & 1094 & 13 & NVSS, Braun et al. (2007) \\
2.695 & 720 & 70 & Haynes et al. (1975) \\
5.000 & 472 & 25 & de Bruyn (1977) \\
6.630 & 360 & 30 & McCutcheon (1973) \\
10.625 & 239 & 10 & Niklas et al. (1995), Israel \& van der Hulst (1983) \\
24.500 & 167 & 10 & this work \\
\hline
\end{tabular}

Table B.6. NGC 3034 .

\begin{tabular}{rrrl}
\hline \hline Frequency & Flux density & Error & Reference \\
\hline 0.327 & 13830 & 690 & Adebahr et al. (2013) \\
0.750 & 10700 & 500 & Kellermann et al. (1969) \\
1.388 & 7805 & 385 & Hummel (1980), Adebahr et al. (2013) \\
2.695 & 5700 & 300 & Kellermann et al. (1969) \\
5.000 & 3900 & 200 & Kellermann et al. (1969) \\
10.700 & 2250 & 60 & Klein et al. (1988) \\
14.700 & 1790 & 40 & Klein et al. (1988) \\
24.500 & 1190 & 30 & Klein et al. (1988) \\
32.000 & 1020 & 60 & Klein et al. (1988) \\
\hline
\end{tabular}

Table B.7. NGC 3079.

\begin{tabular}{rrrl}
\hline \hline Frequency & Flux density & Error & Reference \\
\hline 0.327 & 2440 & 100 & WENSS, this work \\
0.615 & 1740 & 90 & Irwin \& Saikia (2003) \\
1.365 & 800 & 10 & Braun et al. (2007) \\
2.640 & 526 & 30 & this work \\
4.750 & 377 & 20 & Gioia et al. (1982) \\
10.650 & 205 & 11 & Gioia et al. (1982) \\
24.500 & 123 & 8 & this work \\
\hline
\end{tabular}

Table B.8. NGC 3310 .

\begin{tabular}{rrrl}
\hline \hline Frequency & Flux density & Error & Reference \\
\hline 0.327 & 904 & 30 & WENSS, this work \\
0.610 & 650 & 50 & van der Kruit \& de Bruyn (1976) \\
1.433 & 357 & 10 & Condon (1987), Hummel et al. (1985) \\
2.695 & 240 & 30 & van der Kruit \& de Bruyn (1976) \\
4.750 & 146 & 8 & Gioia et al. (1982) \\
10.650 & 100 & 6 & Gioia et al. (1982), Israel \& de Bruyn (1988), Niklas et al. (1995) \\
24.500 & 80 & 5 & this work \\
\hline
\end{tabular}

Table B.9. NGC 4038/39.

\begin{tabular}{rrrl}
\hline \hline Frequency & Flux density & Error & Reference \\
\hline 0.408 & 1250 & 60 & Slee (1995) \\
1.410 & 544 & 30 & van der Hulst (1979), Hummel (1980), this work \\
2.700 & 353 & 30 & de Jong (1967), Tovmassian (1968), Kazès et al. (1970) \\
4.850 & 246 & 17 & Griffith et al. (1994) \\
10.550 & 146 & 7 & Niklas et al. (1995); Chyży \& Beck (2004) re-computed \\
24.500 & 92 & 9 & this work \\
\hline
\end{tabular}


Table B.10. NGC 4449.

\begin{tabular}{rrrl}
\hline \hline Frequency & Flux density & Error & Reference \\
\hline 0.376 & 514 & 30 & Purkayastha (2014) \\
0.609 & 450 & 40 & Klein et al. (1996) \\
1.427 & 278 & 15 & Condon (1987), NVSS, this work Klein \& Emerson (1981) \\
2.695 & 204 & 12 & this work \\
4.875 & 137 & 9 & Klein \& Gräve (1986), Sramek (1975) \\
10.650 & 87 & 5 & Israel \& van der Hulst (1983), Klein \& Gräve (1986), Klein et al. (1996) \\
24.500 & 67 & 10 & Klein \& Gräve (1986) \\
\hline
\end{tabular}

Table B.11. NGC 4490/85.

\begin{tabular}{rrrl}
\hline \hline Frequency & Flux density & Error & Reference \\
\hline 0.327 & 2040 & 50 & WENSS, this work \\
0.408 & 2099 & 50 & Gioia \& Gregorini (1980) \\
1.430 & 835 & 16 & Lequeux (1971), Viallefond et al. (1980), Condon (1987) \\
2.695 & 520 & 50 & Kazès et al. (1970) \\
4.81 & 331 & 13 & Gioia et al. (1982), Nikiel-Wroczyński et al. (2016) \\
6.630 & 262 & 40 & McCutcheon (1973) \\
10.700 & 185 & 27 & Klein \& Emerson (1981) \\
24.500 & 106 & 10 & Klein (1983) \\
\hline
\end{tabular}

Table B.12. NGC 4631.

\begin{tabular}{rrrl}
\hline \hline Frequency & Flux density & Error & Reference \\
\hline 0.327 & 2993 & 110 & Hummel \& Dettmar (1990), WENSS, this work \\
0.419 & 2900 & 71 & Gioia \& Gregorini (1980), Israel \& van der Hulst (1983) \\
0.610 & 2200 & 100 & Ekers \& Sancisi (1977), Werner (1988) \\
0.835 & 2000 & 100 & Israel \& de Bruyn (1988) \\
1.423 & 1282 & 20 & Ekers \& Sancisi (1977), Hummel \& Dettmar (1990), Braun et al. (2007) \\
2.688 & 835 & 32 & Wielebinski \& von Kap-Herr (1977), Werner (1988) \\
4.750 & 544 & 20 & Wielebinski \& von Kap-Herr (1977), Israel \& van der Hulst (1983), Werner (1988) \\
8.35 & 310 & 16 & Mora \& Krause (2013) \\
10.625 & 260 & 10 & Israel \& van der Hulst (1983), Dumke et al. (1995) \\
24.500 & 172 & 15 & this work \\
\hline
\end{tabular}

Table B.13. NGC 5194/5.

\begin{tabular}{rrrl}
\hline \hline Frequency & Flux density & Error & Reference \\
\hline 0.327 & 3812 & 150 & WENSS, this work \\
0.408 & 3640 & 700 & Gioia \& Gregorini (1980) \\
0.610 & 2790 & 200 & Segalovitz (1977) \\
1.365 & 1420 & 10 & Braun et al. (2007) \\
2.695 & 780 & 50 & Klein et al. (1984a) \\
4.750 & 488 & 20 & Gioia et al. (1982), Israel \& van der Hulst (1983), Beck et al. (1987) \\
8.35 & 306 & 26 & Klein \& Emerson (1981) \\
10.700 & 239 & 11 & Israel \& van der Hulst (1983), Klein et al. (1984a) \\
14.700 & 190 & 20 & Klein et al. (1984a) \\
22.800 & 142 & 15 & Klein et al. (1984a) \\
\hline
\end{tabular}

Table B.14. NGC 6052.

\begin{tabular}{rrrl}
\hline \hline Frequency & Flux density & Error & Reference \\
\hline 0.325 & 244 & 20 & Deeg et al. (1993) \\
1.445 & 105 & 5 & Deeg et al. (1993); NVSS, this work \\
4.75 & 41.7 & 0.8 & Klein et al. (1984b), Klein et al. (1991) \\
10.7 & 22.8 & 2.2 & Klein et al. (1991), Heidmann et al. (1982), Maehara et al. (1985) \\
23.7 & 12.5 & 2.1 & Heidmann et al. (1982), Klein et al. (1991) \\
32.0 & $<12$ & & Klein et al. (1991) \\
\hline
\end{tabular}




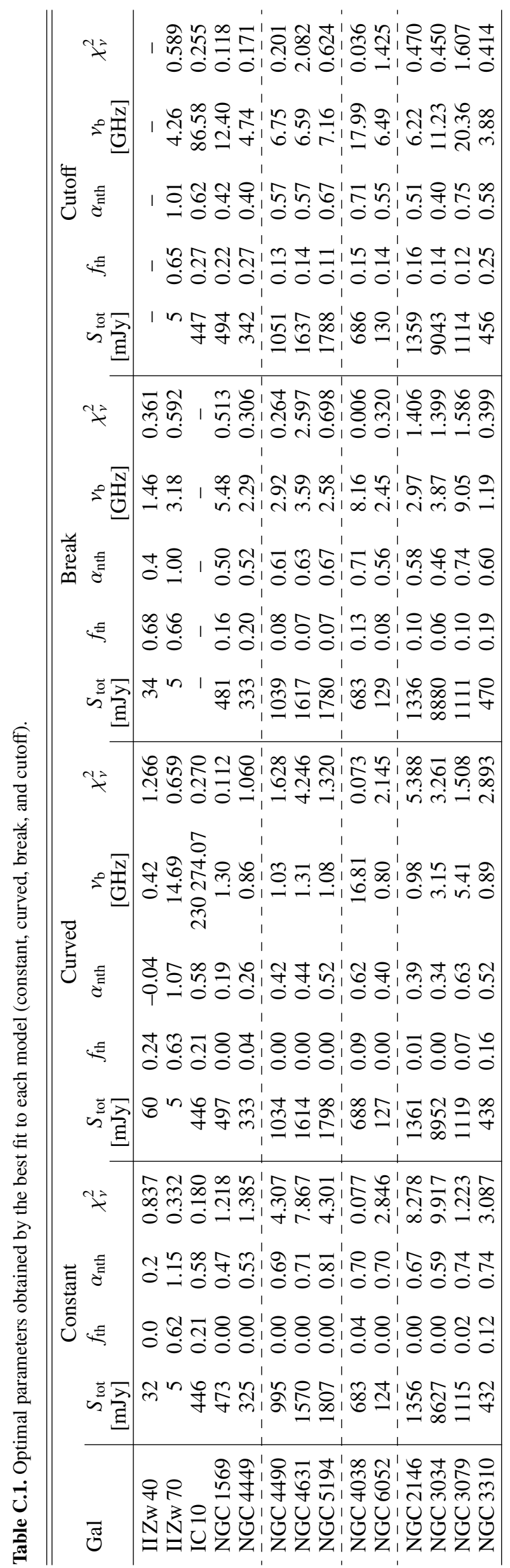

\section{Appendix C: Fit plots}

In this Appendix, we show the best fits of all four models (constant, curved, break, and cutoff) for an easier comparison, along with the parameter spaces and we give the best-fit values for the free parameters in Table C.1. The fitting method has been explained in Sect. 4.2. In short, we tested the four different models presented in Sect. 4.1 for each galaxy (which are represented by the solid red line): (i) simple power-law (i.e. with constant loglog slope); (ii) slightly curved law; (iii) power-law with a break, and (iv) power-law with an exponential decline. The only fixed parameter was the slope of the (optically thin) thermal emission, that is, $\alpha_{\mathrm{th}}=0.1$. The optimal values for the parameters are listed in the lower left part of the figures, along with the reduced chisquare, $\chi_{v}^{2}$.

Then, for a given model, we generate 1000 spectra similar to the observed one. At each frequency, the generated value is allowed to vary within the error of the observational data. This variation follows a Gaussian distribution around the observed data, and the observational error represents $3 \sigma$ of the Gaussian distribution. The 1000 spectra generated randomly this way are represented by faint grey lines in the main plots of the Appendix C. Each generated spectrum is then fitted in the same way as the observational data (see Sect. 4.2) and yields a value for each free parameter. For each model, the distribution of the possible 1000 values taken by the parameters are shown in two parameter space plots under the main plot, allowing an assessment of the robustness of the fits.

In case of IC 10 (IIZw 40), the least-squares minimisation fails for the break model (cutoff model) so that this model cannot be shown.

Apart from the reduced chi-squared, $\chi_{v}^{2}$, an important test for whether or not the fits are physically meaningful is to look at the resulting thermal flux densities obtained at each frequency after subtracting the computed nonthermal flux density from the total (observed). A fit only makes sense if the resulting thermal flux densities so obtained obey to the expected optically thin spectrum with slope -0.10 . This is shown in each diagram by the cyan data points, with the errors taken from those of the measured total flux densities. The resulting slopes are listed in Table C.2.

Table C.2. Slopes of the thermal flux densities.

\begin{tabular}{|c|c|c|c|c|}
\hline Galaxy & Constant & Curved & Break & Cutoff \\
\hline II Zw 40 & -0.87 & -0.09 & -0.09 & - \\
\hline II Zw 70 & -0.10 & -0.10 & -0.10 & -0.10 \\
\hline IC 10 & -0.10 & -0.10 & - & -0.10 \\
\hline NGC 1569 & -0.21 & -0.07 & -0.11 & -0.10 \\
\hline NGC 4449 & -0.29 & -0.10 & -0.11 & -0.11 \\
\hline $\mathrm{NGC} 4490$ & -0.54 & -0.28 & -0.12 & -0.10 \\
\hline NGC 4631 & -0.95 & -0.35 & -0.19 & -0.15 \\
\hline NGC 5194 & -2.12 & -0.50 & -0.13 & -0.11 \\
\hline $\mathrm{NGC} 4038$ & -0.09 & -0.10 & -0.10 & -0.10 \\
\hline NGC 6052 & 0.97 & -0.83 & -0.10 & -0.10 \\
\hline$\overline{\mathrm{NGC}} 214 \overline{4}$ & -0.38 & 0.07 & -0.09 & -0.10 \\
\hline NGC 3034 & -0.88 & -0.75 & -0.25 & -0.11 \\
\hline NGC 3079 & -0.39 & -0.15 & -0.13 & -0.12 \\
\hline NGC 3310 & -0.13 & -0.11 & -0.10 & -0.10 \\
\hline
\end{tabular}

Notes. These linear fits are depicted by solid magenta lines in the figures of the present appendix. 

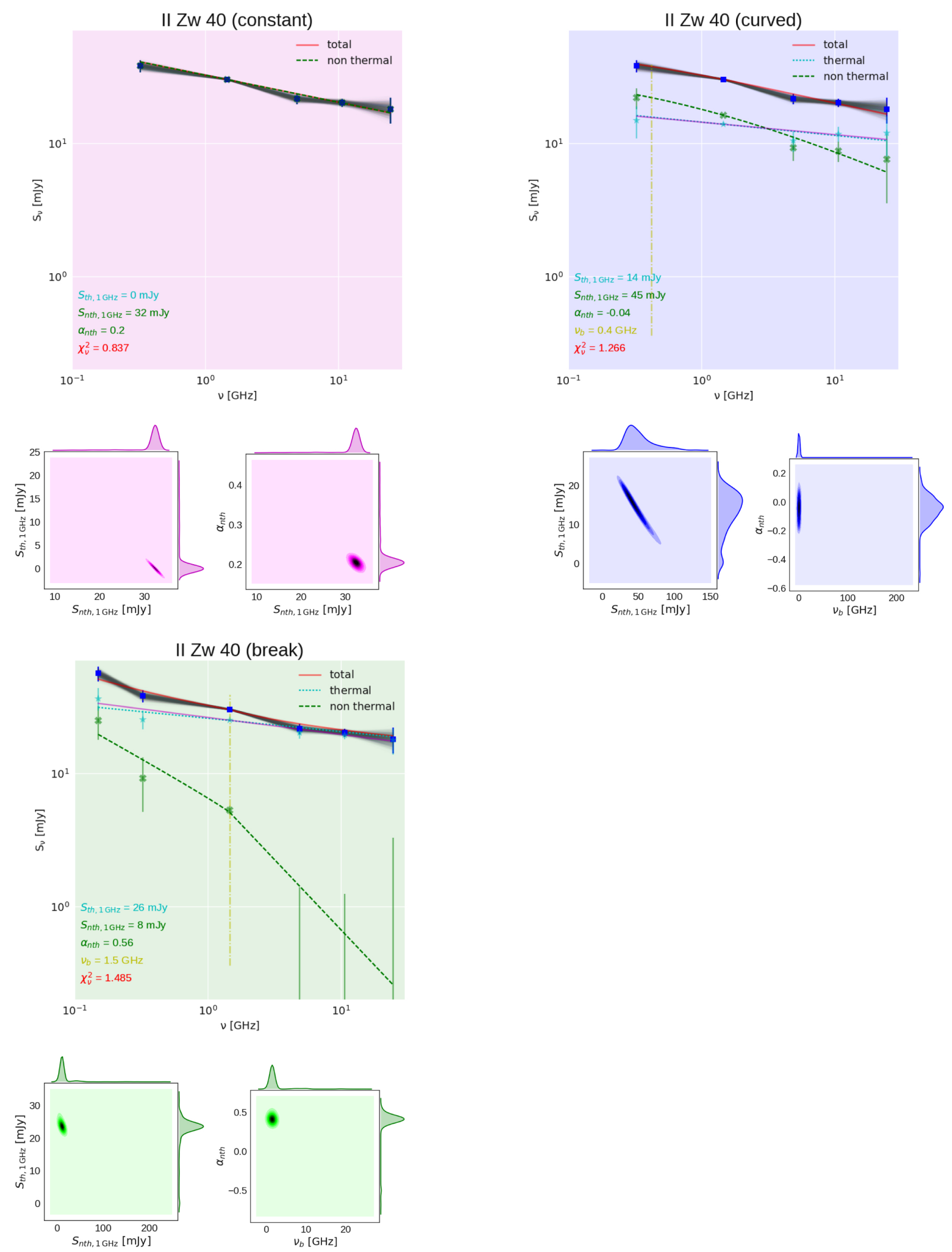

Fig. C.1. Best fit of the radio continuum spectrum is represented by a solid red line. The best parameters are listed in the lower left part of the figure along with the reduced chi-square $\left(\chi_{v}^{2}\right)$. The faint grey lines represent the randomly 1000 spectra generated in the fitting process. The free-free (thermal) and synchrotron (nonthermal) components of the models are depicted by the dotted cyan line and dashed green line, respectively. The green crosses represent the observed nonthermal component (i.e. the modeled thermal component removed from the observational data). The cyan stars delineate the observed thermal component (i.e. the modeled nonthermal component subtracted from the observational data). The thermal component is drawn only if the fitted coefficient, $S_{\mathrm{th}, 1 \mathrm{GHz}}$, is strictly positive. If present (curved, break, and cutoff models), the vertical dash-dotted yellow line depicts the break frequency, $v_{\mathrm{b}}$. The magenta line delineates a linear fit to the thermal flux densities. 
U. Klein et al.: Synchrotron spectra of galaxies
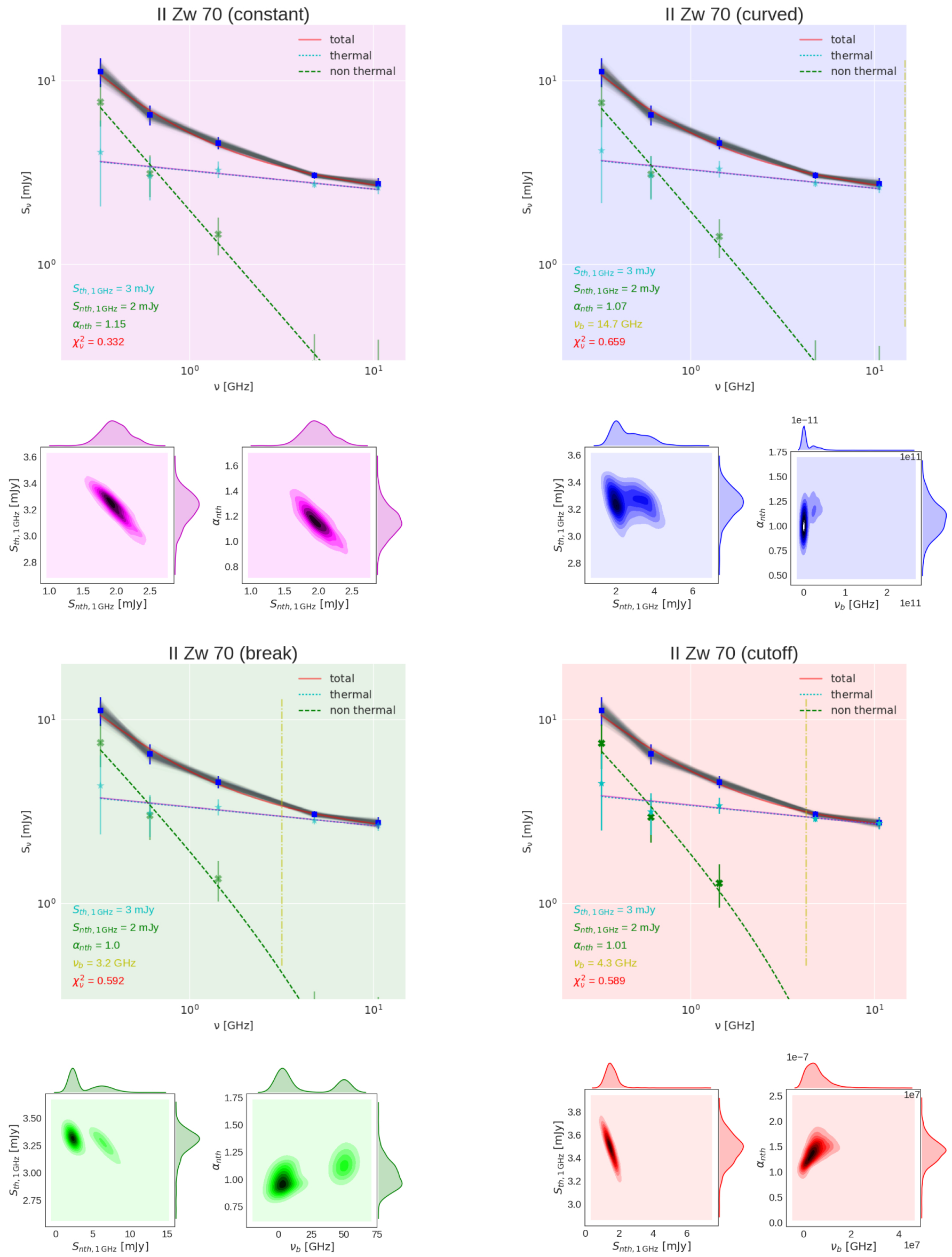

Fig. C.1. continued. 
A\&A 611, A55 (2018)
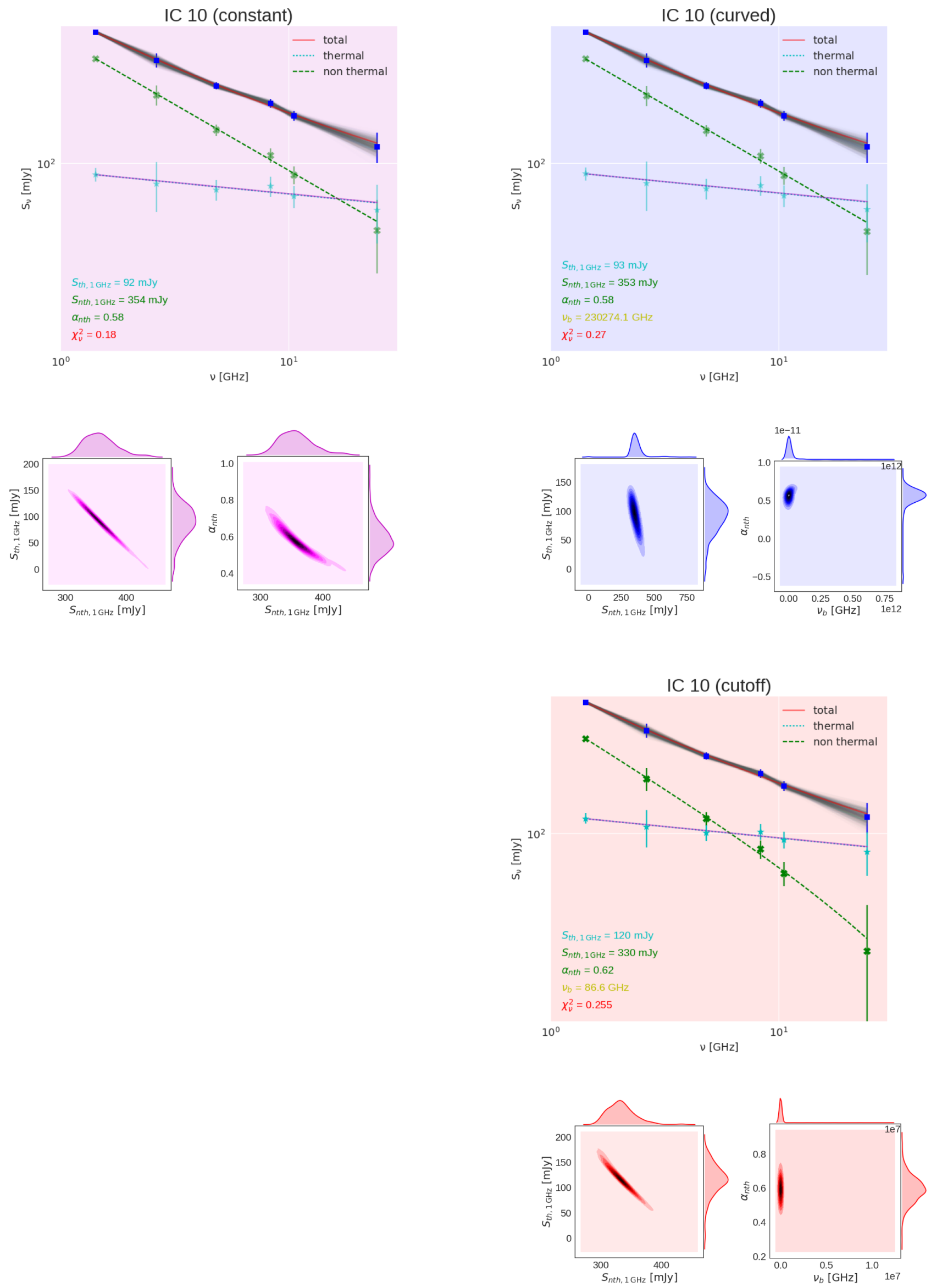

Fig. C.1. continued. 
U. Klein et al.: Synchrotron spectra of galaxies
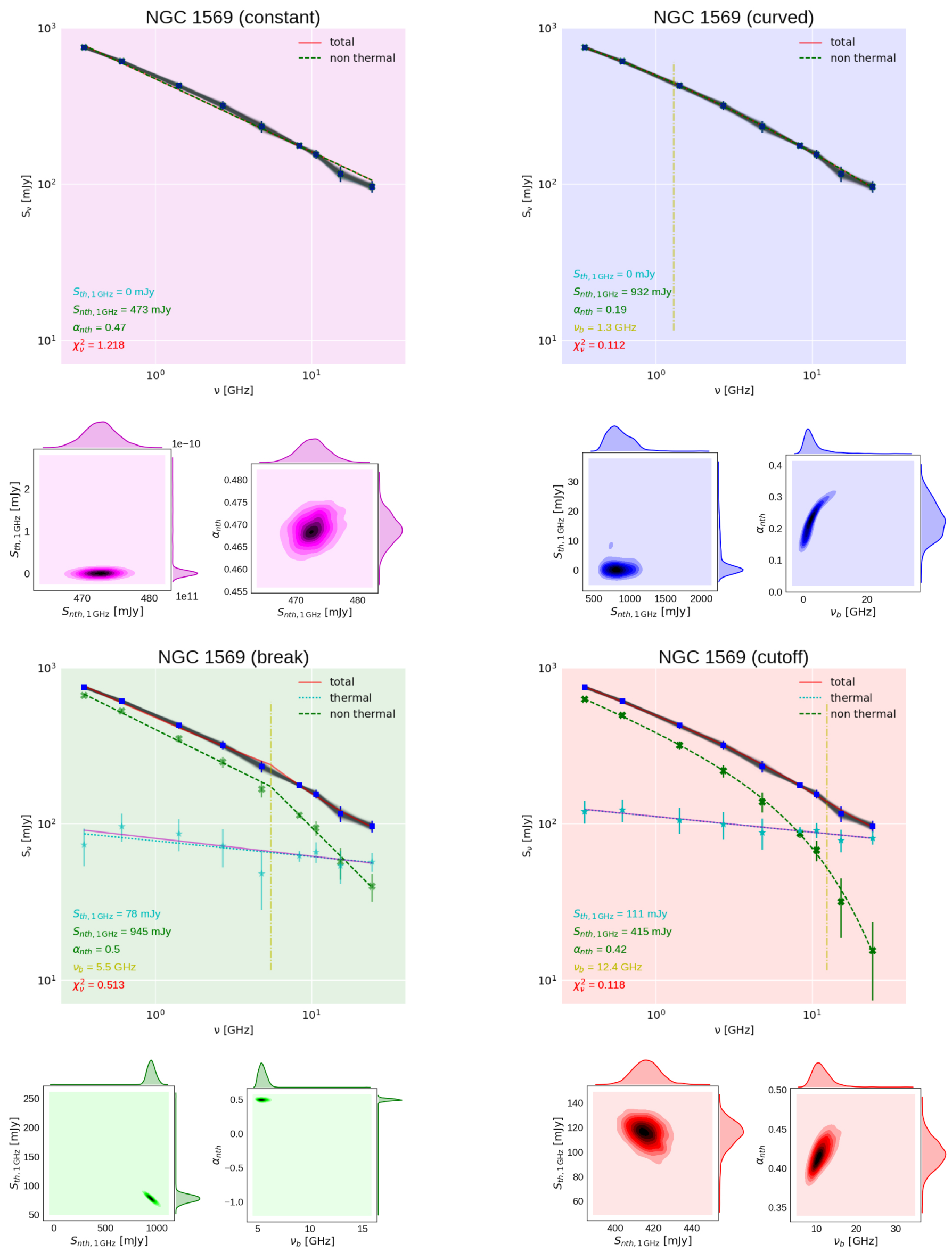

Fig. C.1. continued. 

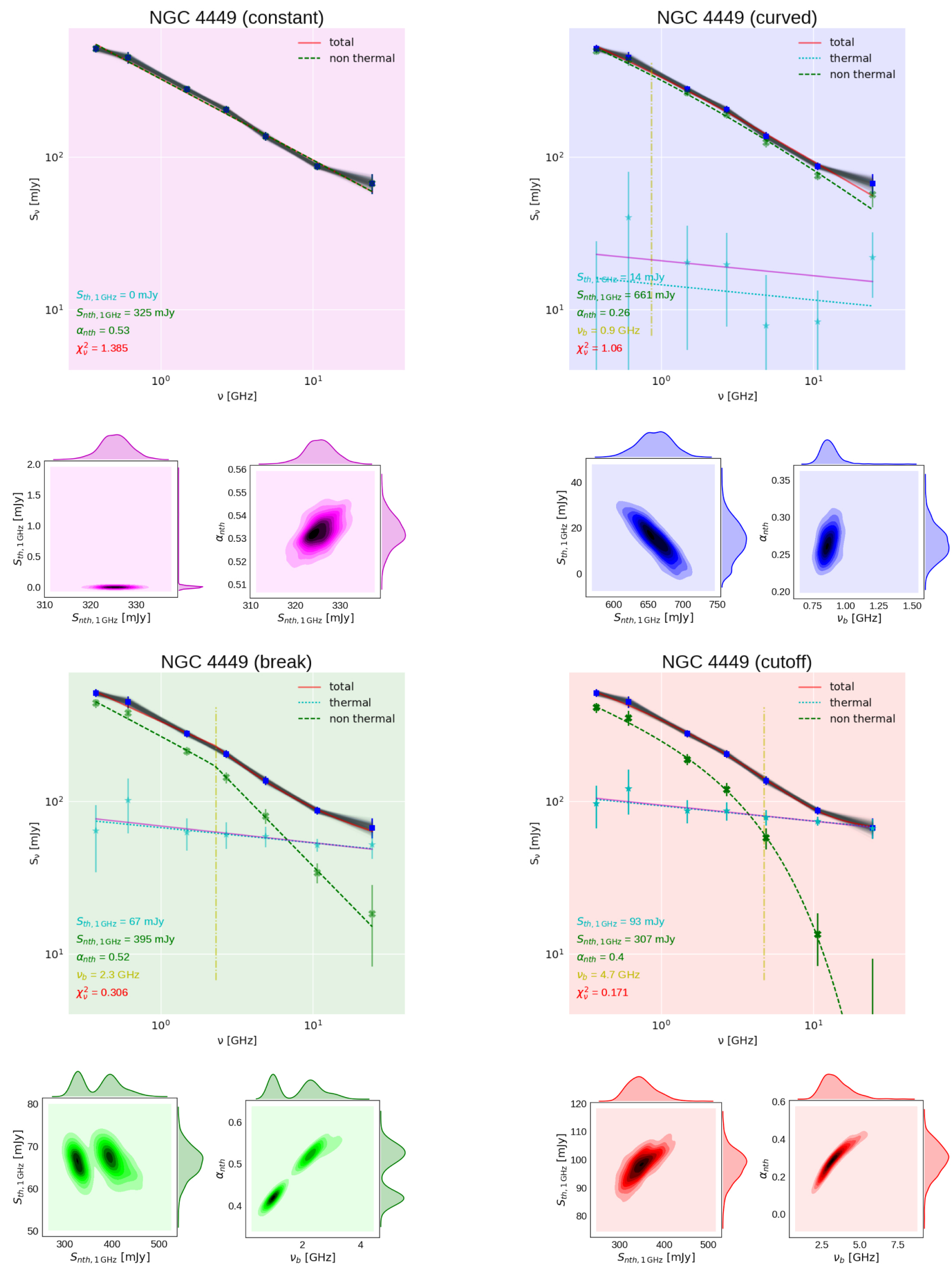

Fig. C.1. continued. 
U. Klein et al.: Synchrotron spectra of galaxies
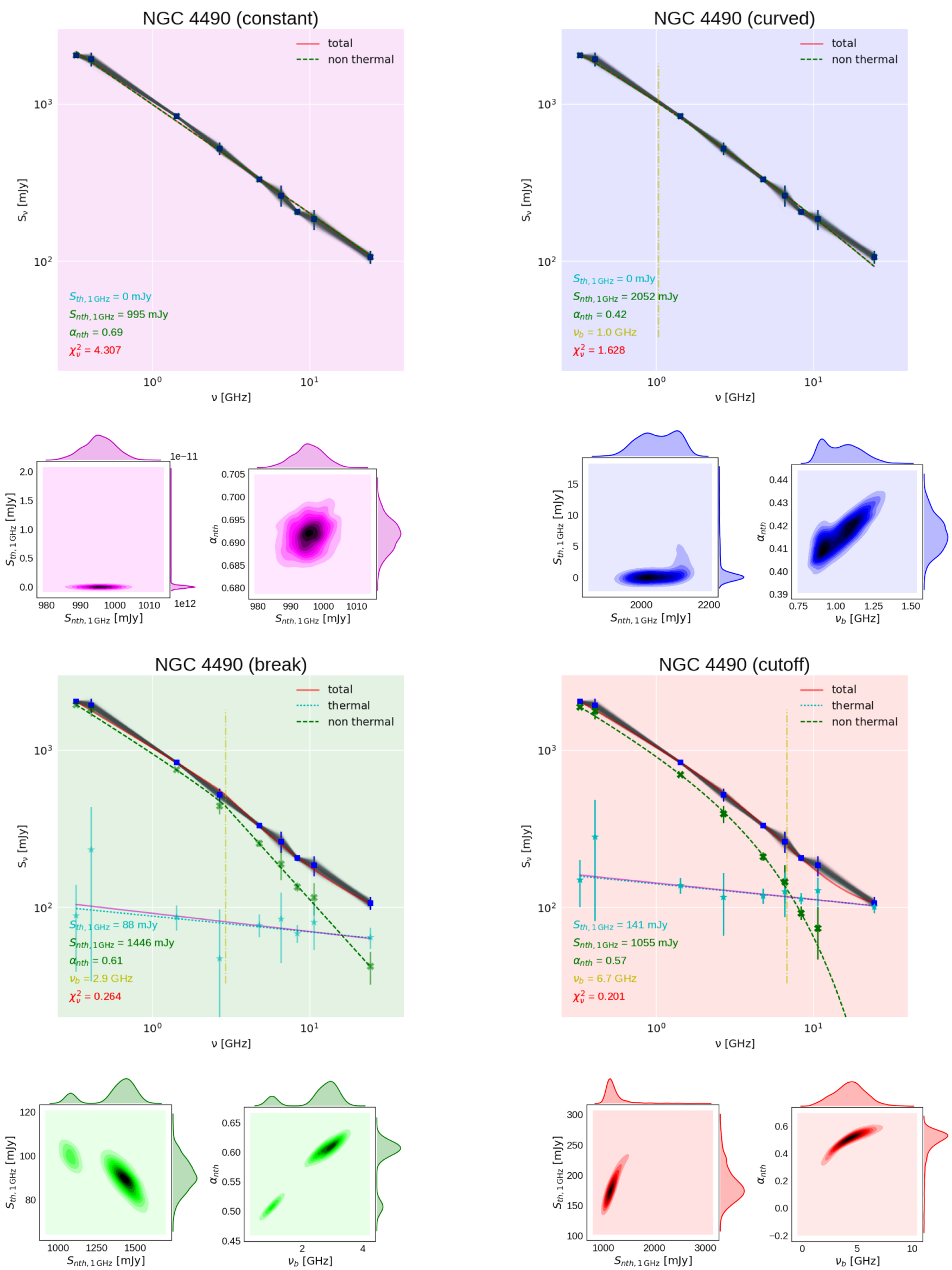

Fig. C.1. continued. 

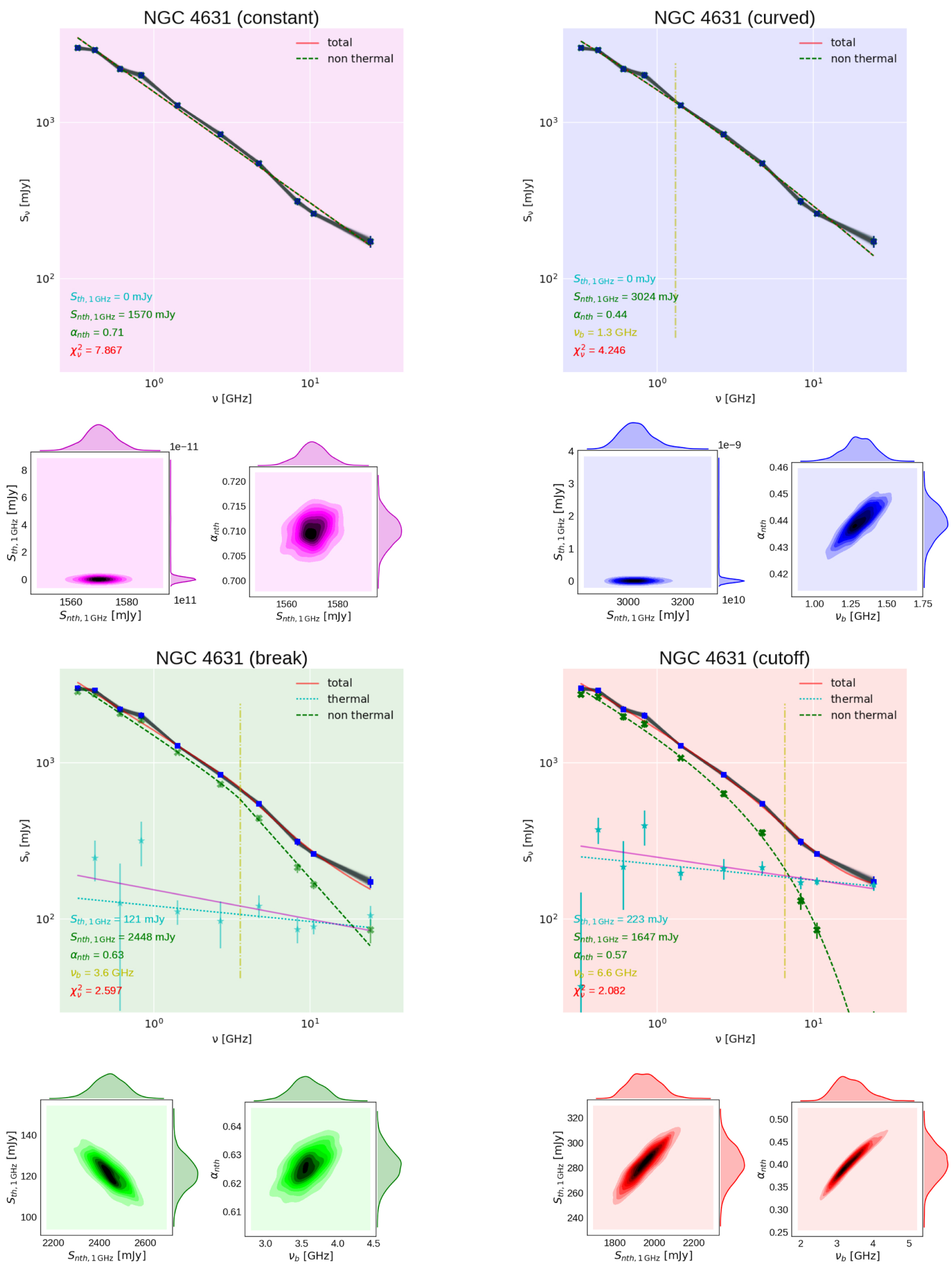

Fig. C.1. continued. 
U. Klein et al.: Synchrotron spectra of galaxies
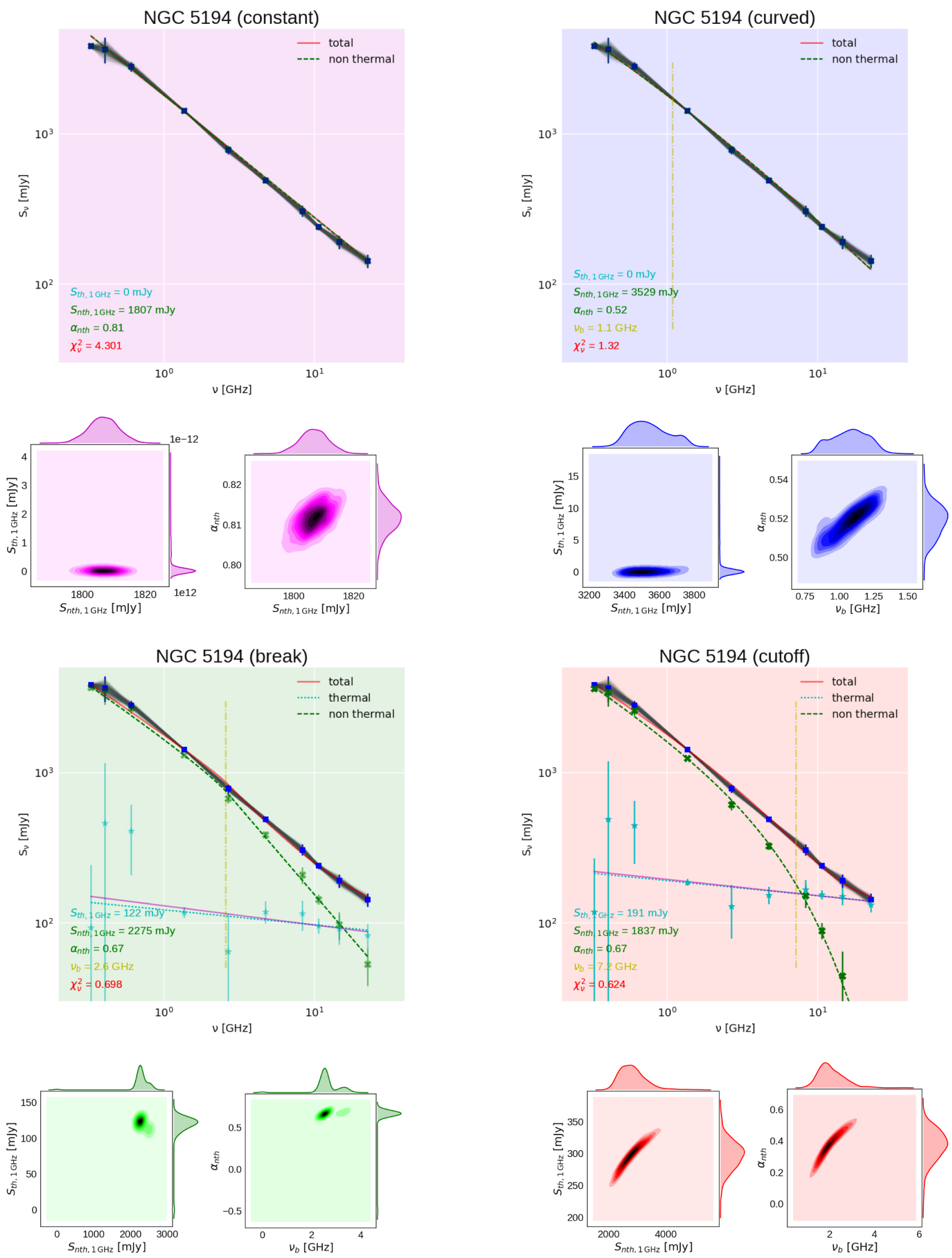

Fig. C.1. continued. 
NGC 4038 (constant)
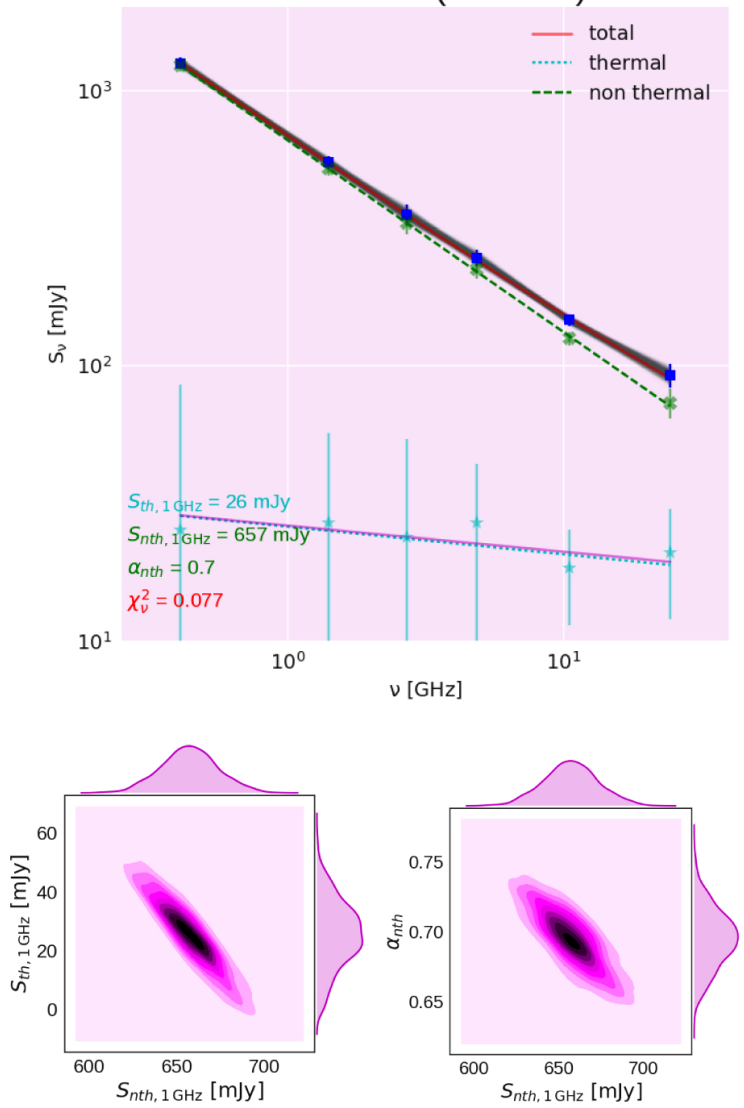

NGC 4038 (break)
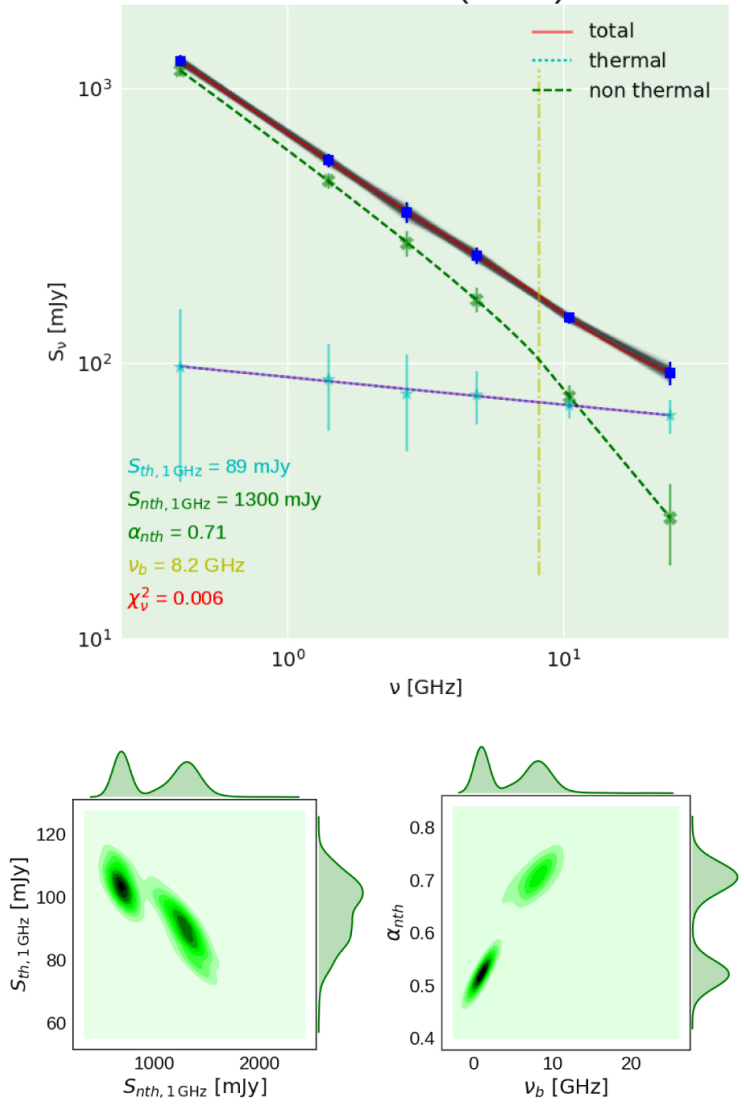

NGC 4038 (curved)
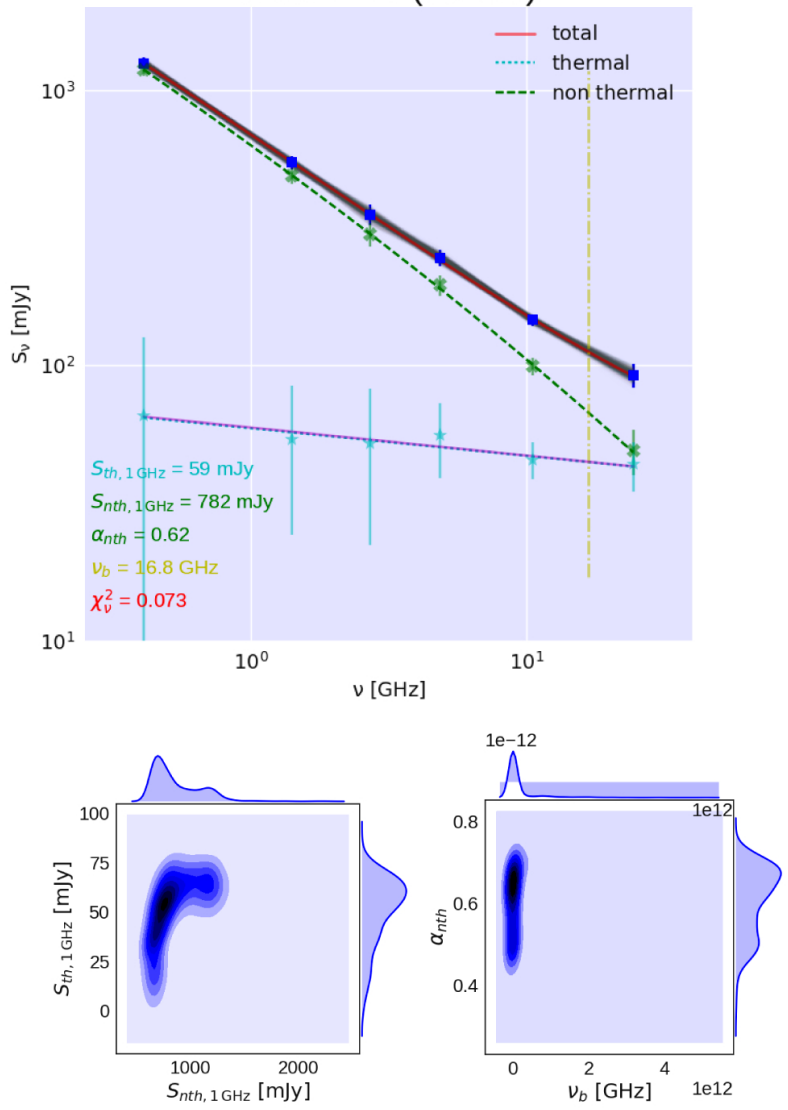

NGC 4038 (cutoff)
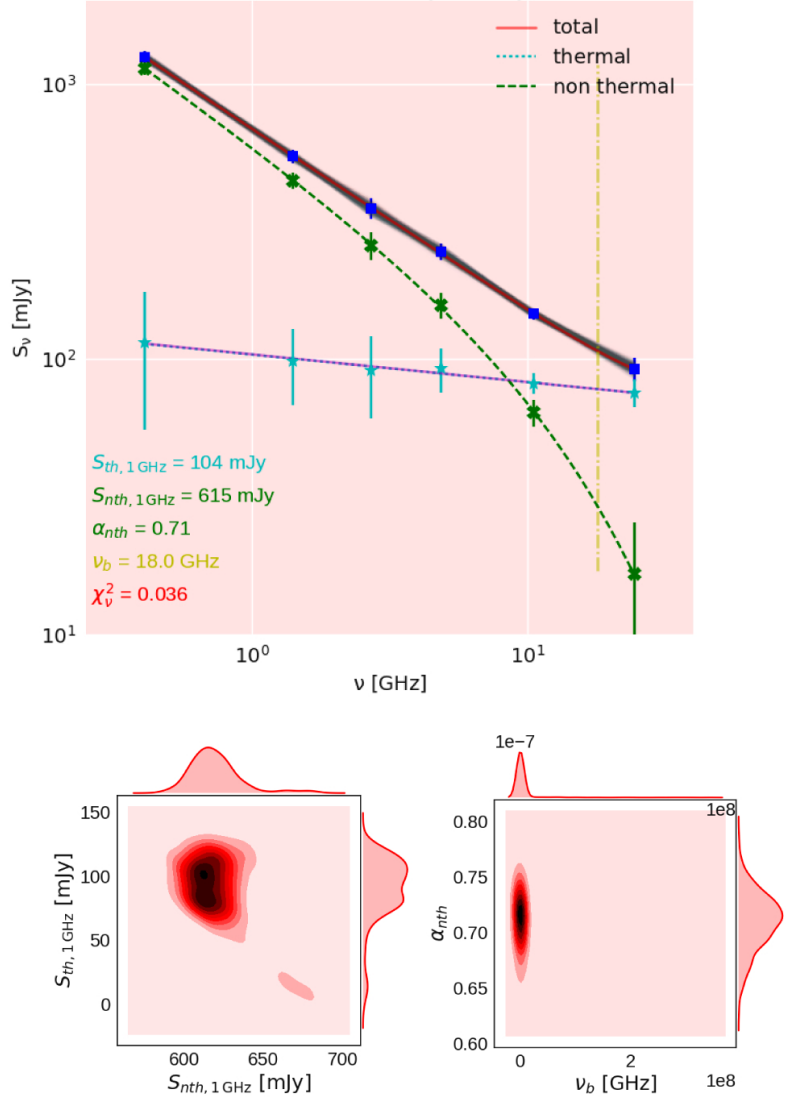

Fig. C.1. continued. 
U. Klein et al.: Synchrotron spectra of galaxies
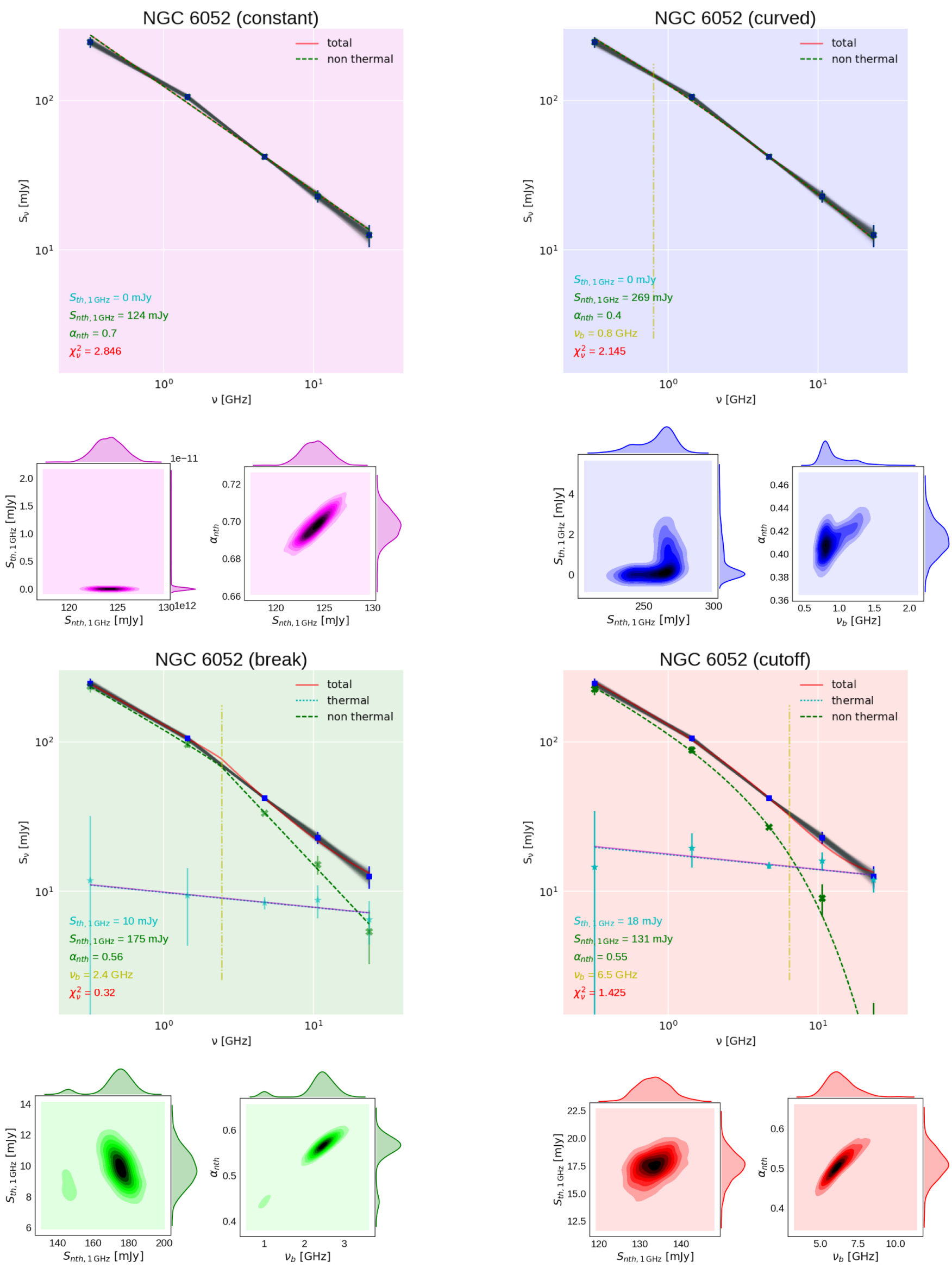

Fig. C.1. continued. 

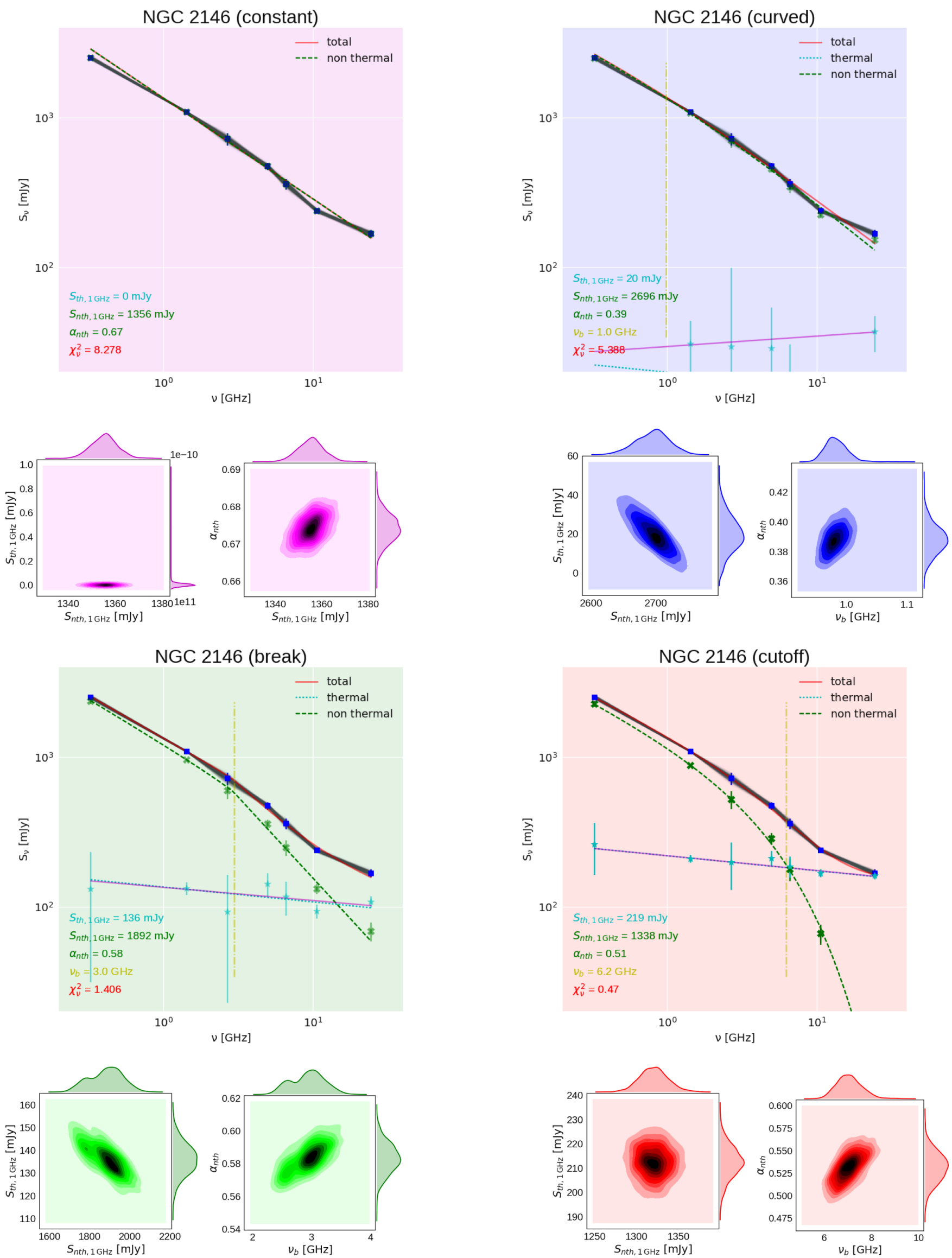

Fig. C.1. continued. 
U. Klein et al.: Synchrotron spectra of galaxies
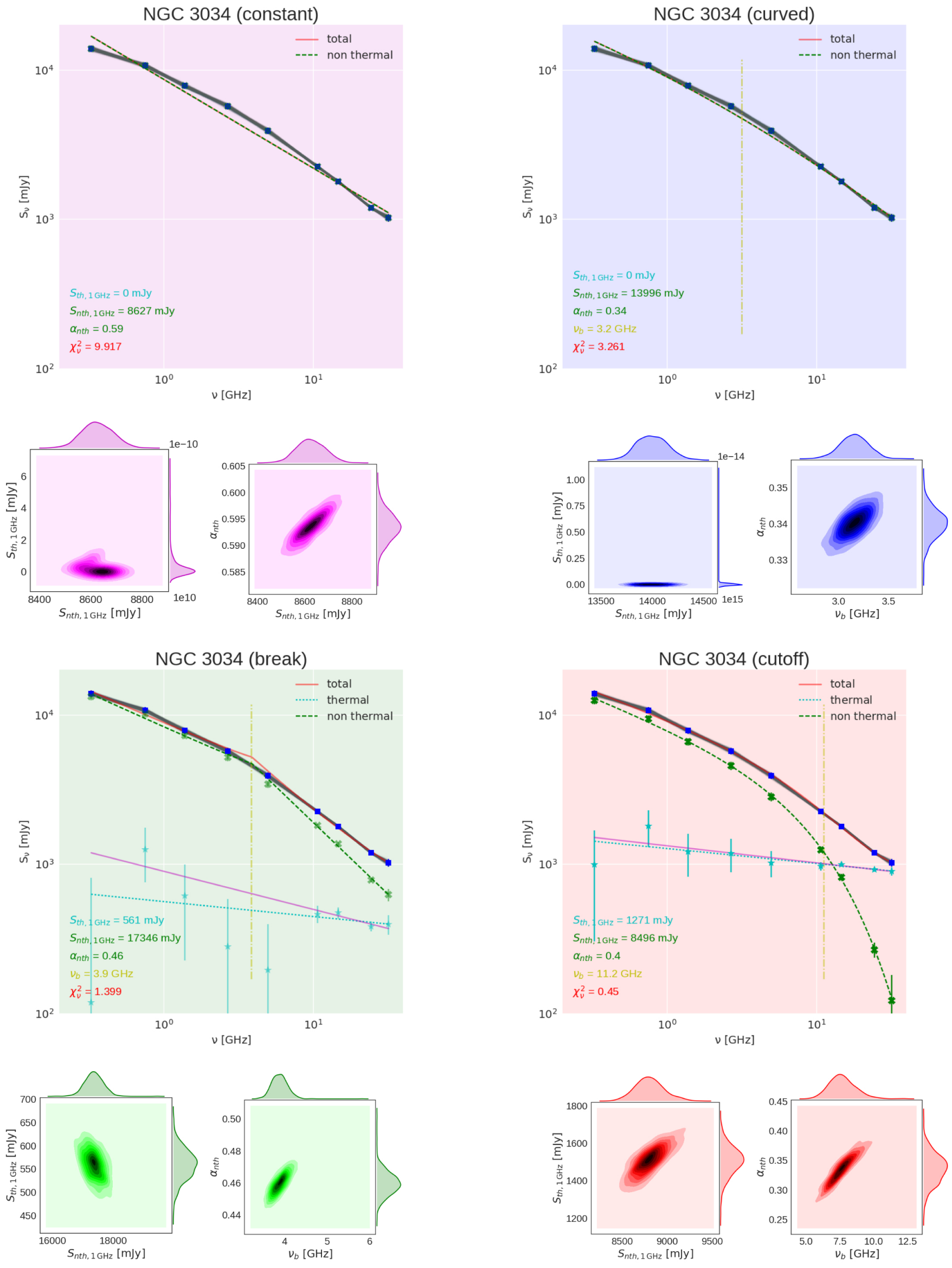

Fig. C.1. continued. 

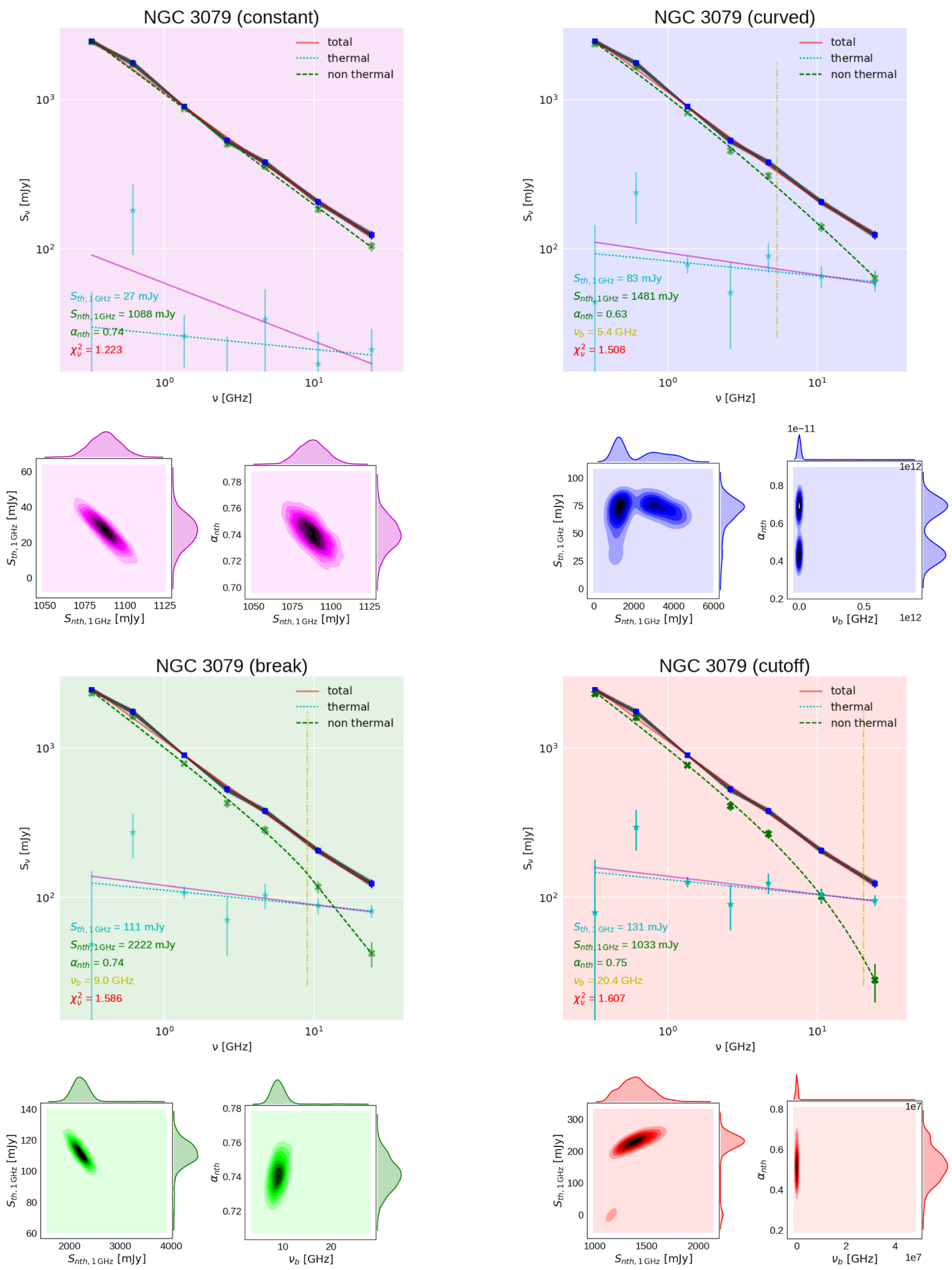

Fig. C.1. continued. 
U. Klein et al.: Synchrotron spectra of galaxies
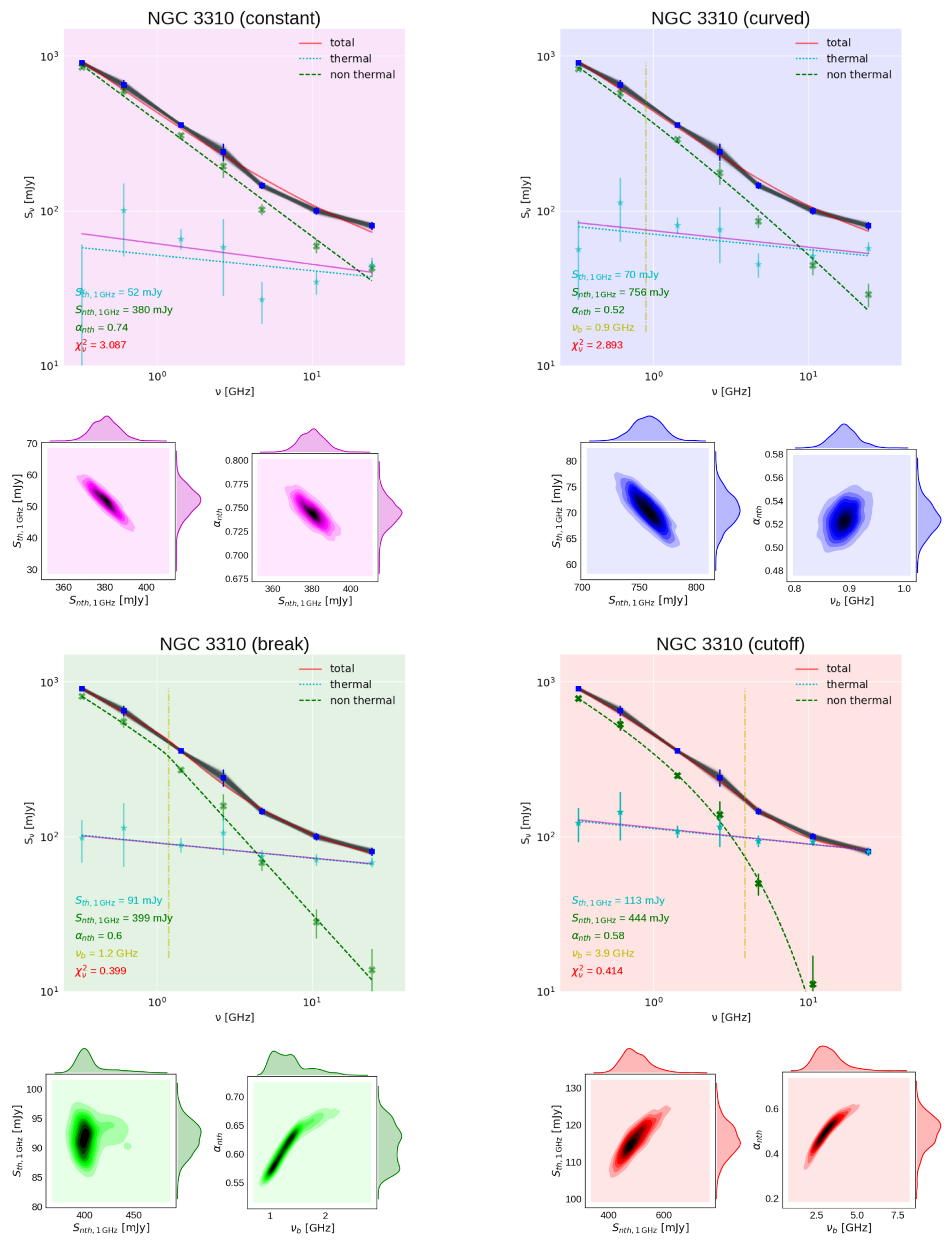

Fig. C.1. continued. 